\title{
Exosomal transfer of miR-25-3p promotes the proliferation and temozolomide resistance of glioblastoma cells by targeting FBXW7
}

\author{
JIANXIN WANG ${ }^{1}$, TIANXIAO $\mathrm{LI}^{2}$ and BIN WANG ${ }^{1}$ \\ Departments of ${ }^{1}$ Neurosurgery, and ${ }^{2}$ Intervention Therapy, Henan Provincial People's Hospital, \\ Henan Provincial Cerebrovascular Hospital, Zhengzhou University People's Hospital, \\ Henan University People's Hospital, Zhengzhou, Henan 450003, P.R. China
}

Received March 24, 2021; Accepted June 24, 2021

DOI: 10.3892/ijo.2021.5244

\begin{abstract}
Intrinsic or acquired resistance to temozolomide (TMZ) is a frequent occurrence in patients with glioblastoma (GBM). Accumulating evidence has indicated that the exosomal transfer of proteins and RNAs may confer TMZ resistance to recipient cells; however, the potential molecular mechanisms are not fully understood. Thus, the aim of the present study was to elucidate the possible role of exosomal microRNAs (miRNAs/miRs) in the acquired resistance to TMZ in GBM. A TMZ-resistant GBM cell line (A172R) was used, and exosomes derived from A172R cells were extracted. Exosomal miR-25-3p was identified as a miRNA associated with TMZ resistance. The potential functions of exosomal miR-25-3p were evaluated by reverse transcription-quantitative PCR, as well as cell viability, colony formation and soft agar assay, flow cytometry, western blot analysis, BrdU incorporation assay, tumor xenograft formation, luciferase reporter assay and RNA immunoprecipitation. It was found that A172R-derived exosomes promoted the proliferation and TMZ resistance of sensitive GBM cells. Moreover, miR-25-3p epxression was upregulated in the exosomes of A172R cells and in serum samples of patients with GBM treated with TMZ. The depletion of exosomal miR-25-3p partially abrogated the effects induced by the transfer of exosomes from A172R cells. By contrast, miR-25-3p overexpression facilitated the proliferation and TMZ resistance of sensitive GBM cells. F-box and WD repeat domain-containing-7 (FBXW7) was identified as a direct target of miR-25-3p. FBXW7 knockdown promoted the proliferation and TMZ resistance of GBM cells. Furthermore, the exosomal transfer of miR-25-3p promoted c-Myc and
\end{abstract}

Correspondence to: Dr Jianxin Wang, Department of Neurosurgery, Henan Provincial People's Hospital, Henan Provincial Cerebrovascular Hospital, Zhengzhou University People's Hospital, Henan University People's Hospital, Zhengzhou, Henan 450003, P.R. China

E-mail: wangjianxin19879@sina.com

Key words: glioblastoma, temozolomide, exosome, miR-25-3p, F-box and WD repeat domain-containing-7 cyclin E expression by downregulating FBXW7. Our results provided a novel insight into exosomal microRNAs in acquired TMZ resistance of GBM cells. Besides, exosomal miR-25-3p might be a potential prognostic marker for GBM patients.

\section{Introduction}

Glioblastoma (GBM) is the most common and lethal form of primary brain tumor affecting humans (1). It originates from astrocytic cells and belongs to grade IV of diffuse glioma according to the WHO classification (1). The incidence rate for GBM is 3.2 per 100, 000 individuals, which ranks first among all brain tumors (2). Notably, GBM comprises up to $45.2 \%$ of malignant primary brain tumors and 54\% of all gliomas (2). It mainly occurs in older patients, and the average age at diagnosis is 64 years. GBM is associated with a very poor prognosis, with a median survival rate as low as 15 months (3). The current standard therapy for patients with GBM includes surgical resection, radiation and temozolomide (TMZ) chemotherapy (4). Despite these aggressive treatments, the majority of patients with GBM develop recurrence within 1 year following treatment, and the 5-year survival rate is $<5 \%$ (5). TMZ, an oral DNA alkylating agent, can inhibit cell cycle progression at the G2/M phase, ultimately leading to apoptosis. Acquired resistance to TMZ often occurs in patients with GBM and this is a predictor of a poor prognosis. Thus, it is crucial to clarify the mechanisms through which GBM cells acquire resistance to TMZ.

Exosomes are extracellular vesicles derived from the endosome. The diameter of exosomes is between 40 to $160 \mathrm{~nm}$, with an average size of almost $100 \mathrm{~nm}$ (6). Exosomes can transfer cytoplasmic or membrane components, such as DNA, RNA, proteins or other bioactive molecules to nearby cells, thus playing a vital role in cell-to-cell communications (7). Tumor-derived exosomes are involved in cancer microenvironment remodeling, angiogenesis, metastasis and drug resistance (8). For example, Skog et al (9) demonstrated that exosomes derived from GBM cells facilitated the tubule formation of endothelial cells and the proliferation of glioma cells. The immunoglobulin superfamily protein, $\mathrm{L} 1$ cell adhesion molecule (L1CAM) has been shown to be associated with the increased growth and metastasis of GBM cells. Pace et al (10) proved that exosomes carrying L1CAM significantly promoted 
the growth and metastasis of glioma cells. Furthermore, Zhang et al (11) found that long non-coding RNA SBF2-AS1 delivered by exosomes from TMZ-resistant cells promoted the tolerance of sensitive GBM cells to TMZ. Although exosomes are crucial for cancer progression and drug resistance in GBM, the underlying molecular mechanism are not yet fully understood.

MicroRNAs (miRNAs/miRs) are a small subset of non-coding RNAs with a length of 19-25 nucleotides. Notably, miRNAs can be delivered from cell to cell via exosomes, thus possessing the function of the genome-wide regulation of gene expression to a larger extent (12). Exosomes are enriched with miRNAs. miRNAs transferred by exosomes are vital for tumor immunity, metastasis and drug resistance (12). For example, as previously demonstrated, miR-1910-3p transferred by exosomes increased the growth, metastasis and autophagy of breast cancer cells by activating NF- $\kappa \mathrm{B}$ and the Wnt/ $\beta$-catenin pathway (13). Exosmal miRNAs also play a vital role in the progression and drug resistance of GBM (14). For example, it was previously demonstrated that the delivery of miR-1238 by exosomes from TMZ-resistant GBM cells facilitated TMZ tolerance to other GBM cells by regulating the CAV1/EGFR axis (15).

$\mathrm{miR}-25-3 \mathrm{p}$ is well-characterized in cancers. Of note, miR-25-3p is upregulated and plays an oncogenic role in various types of cancer, such as pancreatic (16), breast (17), esophageal (18), gastric cancer (19) and osteosarcoma (20). The present study investigated exosomal miRNAs from TMZ-resistant GBM cells. Exosomal miRNAs were evaluated by high-throughput RNA sequencing. miR-25-3p was identified as an exosomal miRNA associated with TMZ resistance. The possible role of exosomal miR-25-3p was thus evaluated in in vitro and in vivo experiments. F-box and WD repeat domain-containing-7 (FBXW7) was proven to be a direct target of miR-25-3p. The exosomal transfer of miR-25-3p regulated the proliferation and TMZ resistance of GBM cells by inhibiting FBXW7, thus promoting the expression of oncoproteins, such as c-Myc and cyclin E. The findings of the present study provide insight into the role of exosomal miRNAs in the acquired resistance of GBM cells to TMZ.

\section{Materials and methods}

Patient samples. All patients and healthy donors signed written informed consent forms. The collection and use of serum samples were approved by the Ethics Committee of Henan Provincial People's Hospital [SYXK (Yu) 2018-0004]. Serum samples from 42 patients with GBM treated with TMZ, 34 untreated patients with GBM and 15 healthy donors were obtained at Henan Provincial People's Hospital from June, 2018 to July, 2019. The demographic and clinicopathological data of the patients with GBM were extracted from the hospital database. The patients and healthy donors did not exhibit any significant differences as regards age, sex or nationality (Table I). All patients in the present study were Chinese. The serum samples were maintained at $-80^{\circ} \mathrm{C}$ until use.

Cells, cell culture and reagents. 293T cells, and the GBM cells, U87 (ATCC \#HTB-14) and A172 (ATCC \#CRL-1620), were obtained from the American Type Culture Collection (ATCC). The U87 cell line used in the present study is considered is probably a GBM cell line; however, its true origin is unknown. All cell lines were authenticated by STR profiling. All cells were maintained in Dulbecco's modified Eagle's medium (DMEM, HyClone; Cytiva). The culture medium was supplemented with $10 \%$ fetal bovine serum (HyClone; Cytiva), $100 \mathrm{U} / \mathrm{ml}$ penicillin and $100 \mu \mathrm{g} / \mathrm{ml}$ streptomycin. The cells were cultured at $37^{\circ} \mathrm{C}$ with $5 \% \mathrm{CO}_{2}$ under a humidified condition. The A172R cell line was constructed by treating the A172 cells with stepwise increasing concentrations of TMZ (20 to $200 \mu \mathrm{M}$ ) for 4 months until a stable resistant phenotype was achieved (data not shown). These TMZ-resistant A172 cell populations were dispersed as single cells in 96-well plates and exposed to $200 \mu \mathrm{M} \mathrm{TMZ}$ to obtain TMZ-resistant subclones. Finally, 5 subclones were examined and they exhibited a similar morphology and growth rates. Thus, one subclone of these was designated as A172R cells and subjected to miRNA sequencing analysis. TMZ (\#S1237) was purchased from Selleck Chemicals. Dimethyl sulfoxide (DMSO) was used to dissolve TMZ.

Plasmid constructs, lentiviral packaging and infection. Anti-miR-25-3p was constructed by inserting 8 repeats of antisense miR-25-3p (5'-TCAGACCGAGACAAGTGCAAT G-3') into the pGLV3/H1/GFP vector (Shanghai GenePharma Co., Ltd.) as previously described (21). Anti-miR-ctrl was constructed by cloning 8 repeats of artificial miRNA (5'-AAG TTTTCAGAAAGCTAACA-3') into the pGLV3/H1/GFP vector. Ectopic miR-25-3p expression was achieved by inserting the miR-25-3p mature sequence (5'-CATTGCACTTGTCTCGGT CTGA-3') into the pCMV-MIR vector (\#PCMVMIR; OriGene Technologies, Inc.). Empty pCMV-MIR vector was regarded as miR-ctrl. Single-guide RNA (sgRNA) plasmids targeting human FBXW7 (sgFBXW7-1 and sgFBXW7-2) were constructed by cloning these sgRNAs into lentiCRISPRv2 plasmid (plasmid\#52961; Addgene, Inc.). A non-targeting sequence was inserted into the lentiCRISPRv2 vector as a sgNC control. The DNA sequences for sgRNAs were as follows: sgFBXW7-1, 5'-ACCTACTCTAAACCATGGCT-3'; sgFBXW7-2, 5'-AGC ACAGAATTGATACTAAC-3'; sgNC, 5'-ACGGAGGCTAAG CGTCGCAA-3'. miRNA antagomirs (100 nM) for miR-670-5p, miR-3194-5p, miR-211-5p, miR-4423-5p, miR-25-3p, miR-599, miR-486-5p, miR-221-3p, miR-10a-5p and miR-155-5p, and an antagomir control were synthesized by Guangzhou RiboBio Co., Ltd. and introduced into GBM cells $\left(2 \times 10^{6}\right)$ using Lipofectamine $3000^{\circledR}$ (Invitrogen; Thermo Fisher Scientific, Inc.). A lentiviral package (3rd generation system) was conducted by introducing lentiviral vectors and helper virus packaging plasmids (pCMVAR8.9 and pHCMV-VSV-G; 10:10:1) into 293T cells using Lipofectamine $3000^{\circledR}$ (Invitrogen; Thermo Fisher Scientific, Inc.) at $37^{\circ} \mathrm{C}$ overnight. Culture medium containing virus was collected at 24, 48 and $72 \mathrm{~h}$ following transfection, and then maintained at $-80^{\circ} \mathrm{C}$ until further use. Lentivirus infection was achieved by incubating $\left(1 \times 10^{6}\right)$ cells with $1 \mathrm{ml}$ virus-containing medium $\left(\sim 5 \times 10^{7}\right.$ viral particles $)$ and $8 \mu \mathrm{g} / \mathrm{ml}$ polybrene (Sigma-Aldrich; Merck KGaA) overnight. The cells were then used in subsequent experiments at $24 \mathrm{~h}$ post-infection. Transient transfection was conducted according to the standard protocol provided with Lipofectamine $3000^{\circledR}$ (Invitrogen; Thermo Fisher Scientific, Inc.). Cells were introduced with the indicated plasmids at $37^{\circ} \mathrm{C}$ overnight and used in subsequent experiments at $24 \mathrm{~h}$ post-transfection. 
Table I. Association between serum miR-25-3p levels and clinicopathological characteristics of patients with glioblastoma.

\begin{tabular}{|c|c|c|c|c|}
\hline \multirow[b]{2}{*}{ Characteristics } & \multirow{2}{*}{$\begin{array}{c}\text { No. of } \\
\text { patients }\end{array}$} & \multicolumn{2}{|c|}{ Serum miR-25-3p level } & \multirow[b]{2}{*}{ P-value } \\
\hline & & Low & High & \\
\hline Age (years) & & & & 0.845 \\
\hline$\leq 60$ & 35 & 16 & 19 & \\
\hline$>60$ & 41 & 23 & 18 & \\
\hline Sex & & & & 0.895 \\
\hline Male & 39 & 18 & 21 & \\
\hline Female & 37 & 21 & 16 & \\
\hline Tumor size (mm) & & & & 0.029 \\
\hline$\geq 30$ & 49 & 19 & 30 & \\
\hline$<30$ & 27 & 20 & 7 & \\
\hline Tumor location & & & & 0.748 \\
\hline Supratentorial & 53 & 28 & 25 & \\
\hline Infratentorial & 23 & 11 & 12 & \\
\hline \multicolumn{5}{|c|}{ Temozolomide response } \\
\hline Sensitive & 29 & 23 & 6 & 0.001 \\
\hline Resistant & 47 & 16 & 31 & \\
\hline KPS score & & & & 0.338 \\
\hline$<80$ & 51 & 24 & 27 & \\
\hline$\geq 80$ & 25 & 15 & 10 & \\
\hline
\end{tabular}

Values in bold font indicate statistically significant differences $(\mathrm{P}<0.05)$. KPS score, Karnofsky performance score.

Exosomal isolation. Exosomes were extracted from the culture medium of GBM cells as previously described (15). Briefly, serum samples or culture media were centrifuged at $10,000 \mathrm{x} \mathrm{g}$ for $30 \mathrm{~min}$ at $4^{\circ} \mathrm{C}$ to remove large microvesicles. The supernatants were then filtered using a $0.22-\mu \mathrm{m}$ filter, and centrifuged at $100,000 \mathrm{x}$ for $3 \mathrm{~h}$ at $4^{\circ} \mathrm{C}$. The pellets were dissolved and centrifuged again at $100,000 \times \mathrm{g}$ for $3 \mathrm{~h}$ at $4^{\circ} \mathrm{C}$. Exosomes were dissolved in PBS, and maintained at $-80^{\circ} \mathrm{C}$ until further use. A BCA kit (Thermo Fisher Scientific, Inc.) was used to examine the concentration of exosomes.

Cell viability assay. Cell viability was determined using a Cell Counting Kit-8 (CCK-8; Beyotime Institute of Biotechnology, Inc.) as per the manufacturer's protocol. At each time point, the cells were incubated with $10 \mu \mathrm{l}$ CCK- 8 solution per well in 96 -well plates at $37^{\circ} \mathrm{C}$ for $3 \mathrm{~h}$. The absorbance at $450 \mathrm{~nm}$ was detected using a microplate reader (Multiskan SkyHigh; Thermo Fisher Scientific, Inc.).

Exosome internalization assay. The PKH67 Green Fluorescent Cell Linker kit (Sigma-Aldrich, Merck KGaA) was used for the labeling of exosomes according to the manufacturer's instructions. Cells $\left(1 \times 10^{6}\right)$ were stained with PKH67-labeled exosomes $(2 \mu \mathrm{g})$ for $2 \mathrm{~h}$ at $37^{\circ} \mathrm{C}$ in exosome-free medium. The cells were fixed by $4 \% \mathrm{PFA}$ for $20 \mathrm{~min}$ at $4^{\circ} \mathrm{C}$, then washed with cold PBS on ice for three times. The nuclei were then stained with DAPI (Sigma-Aldrich, Merck KGaA) for $15 \mathrm{~min}$ at $4^{\circ} \mathrm{C}$. The cells were kept on ice and viewed under a laser scanning microscope (Zeiss AG).
Colony formation and soft agar assay. For colony formation assay, cells were seeded $(3,000 /$ well) in 6-well plates for 3 weeks until colonies reached an appropriate diameter ( $\geq 50 \mu \mathrm{m}$ ). The colonies were then fixed with $4 \%$ paraformaldehyde for $15 \mathrm{~min}$ at room temperature and then incubated with $0.5 \%$ crystal violet (Beyotime Institute of Biotechnology) for $1 \mathrm{~h}$ at room temperature. For soft agar assay, cells were seeded $(8,000$ cells/well) in $0.4 \%$ top agar in 6 -well plates. The colonies were allowed to grow for 3 weeks. The colonies were then incubated with $1 \mathrm{mg} / \mathrm{ml}$ thiazolyl blue tetrazolium bromide (MTT; Sigma-Aldrich; Merck KGaA) at $37^{\circ} \mathrm{C}$ for $3 \mathrm{~h}$. Images for colonies were obtained using a scanner (TMA 1600III; Shanghai Microtek Technology Co., Ltd.).

Western blot analysis. Cells were digested using RIPA buffer (Beyotime Institute of Biotechnology, Inc.) supplemented with protease inhibitors (Sigma-Aldrich; Merck KGaA). The protein concentration was examined using a BCA kit (Thermo Fisher Scientific, Inc.). Proteins $(25 \mu \mathrm{g})$ were separated by 10 or $15 \%$ SDS-PAGE, then transferred onto nitrogen membranes. Blocking was performed by incubating the membranes with $5 \%$ non-fat milk for $1 \mathrm{~h}$ at room temperature. Target proteins were detected by staining with specific primary antibodies at $4^{\circ} \mathrm{C}$ overnight and corresponding secondary antibody at room temperature for $1 \mathrm{~h}$. The protein signals were determined using an ECL plus kit (\#32132X3; Thermo Fisher Scientific, Inc.) on a ChemiDoc Touch Imaging System (Bio-Rad Laboratories, Inc.). Relative protein quantification was performed using Image Quant TL 8.1 (GE Healthcare; Cytiva) software. The 
specific antibodies used in the present study were as follows: Cleaved PARP (Asp214) rabbit monoclonal antibody (mAb; 1:1,000; cat. no. 5625), full-length PARP rabbit mAb $(1: 1,000$; cat. no. 9532), GAPDH (D16H11) rabbit mAb (1:1,000; cat. no. 5174) (all from Cell Signaling Technology, Inc.), anti-FBXW7 (1:500; cat. no. ab109617, Abcam), anti-Ago (1:500; cat. no. ab279392, Abcam), c-Myc (E5Q6W) rabbit mAb (1:1,000; cat. no. 18583), cyclin E1 rabbit mAb (1:1,000; cat. no. 20808), cleaved caspase-3 (Asp175) rabbit mAb (1:1,000; cat. no. 9664), caspase-3 rabbit antibody (1:1,000; cat. no. 9662) and anti-rabbit IgG HRP-linked antibody (1:4,000; cat. no. 7074) (all from Cell Signaling Technology, Inc.). The anti-CD63 rabbit $\mathrm{mAb}(1: 1,000$, cat. no. ab134045) and anti-CD81 rabbit mAb (1:1,000, ab219209) were purchased from Abcam.

Flow cytometry. Cells were dispersed as single cells using $0.05 \%$ trypsin. Subsequently, $1 \times 10^{6}$ cells were collected and suspended in $300 \mu \mathrm{l}$ PBS. Cell apoptosis was evaluated by staining the cells using the Annexin V-FITC detection kit (Beyotime Instittue of Biotechnology, Inc.) as per the manufacturer's protocol. Propidium iodide (PI) was used to stain the nuclei. A Guava EasyCyte 6HT-2L flow cytometer (EMD Millipore) was used to determine apoptotic cells. The results of flow cytometry were analyzed using FlowJo 7.6 software (BD Biosciences).

Reverse transcription-quantitative polymerase chain reaction (RT-qPCR). Total RNA was extracted using TRIzol reagent (Invitrogen; Thermo Fisher Scientific, Inc.). The level of miR-25-3p was measured using TaqMan fast advanced master mix (Applied Biosystems; Thermo Fisher Scientific, Inc.). Complementary DNA was synthesized using the RevertAid First Strand cDNA Synthesis kit (Thermo Fisher Scientific, Inc.). The amplification reactions for RT-PCR were as follows: $25^{\circ} \mathrm{C}$ for $10 \mathrm{~min}, 42^{\circ} \mathrm{C}$ for $15 \mathrm{~min}$ and $85^{\circ} \mathrm{C}$ for $50 \mathrm{sec}$. The level of FBXW7 was detected using Express $\mathrm{SYBR}^{\circledR}$-GreenER ${ }^{\mathrm{TM}}$ qPCR SuperMix (Thermo Fisher Scientific, Inc.). qPCR was performed on the ABI 7900 Real-Time PCR system (Applied Biosystems; Thermo Fisher Scientific, Inc.). The amplification reactions for qPCR were as follows: $94^{\circ} \mathrm{C}$ for $10 \mathrm{~min}, 29$ cycles of $94^{\circ} \mathrm{C}$ for $30 \mathrm{sec}, 55^{\circ} \mathrm{C}$ for $30 \mathrm{sec}$, and $72^{\circ} \mathrm{C}$ for $30 \mathrm{sec}$. GAPDH and U6 were used as internal controls. Relative RNA expression was measured using the $2^{-\Delta \Delta \mathrm{Cq}}$ method (22). The primer sequences used were as follows: FBXW7 forward, 5'-TGGTCAGCA GTCACAGGCAAAT-3' and reverse, 5'-GCATACAACGCA CAGTGGAAGT-3'; GAPDH forward, 5'-TGCACCACCAAC TGCTTAGC-3' and reverse, 5'-GGCATGGACTGTGGTCAT GAG-3'; and U6 forward, 5'- CGCTTCGGCAGCACATATACT A-3' and reverse, 5'-CGCTTCACGAATTTGCGTGTCA-3'.

BrdU incorporation assay. Cells were grown on coverslips. The cells were then stained with $10 \mu \mathrm{M}$ BrdU (Beyotime Institute of Biotechnology) for $4 \mathrm{~h}$ at $37^{\circ} \mathrm{C}$ and were then incubated with BrdU mouse mAb (1:300; cat. no. 5292; Cell Signaling Technology, Inc.) for $1 \mathrm{~h}$ at room temperature. The cells were then stained with anti-mouse IgG Alexa Fluor 488 (1:300; cat. no. 4408, Cell Signaling Technology, Inc.) for $1 \mathrm{~h}$ at room temperature avoiding light. The nuclei were stained with DAPI for $5 \mathrm{~min}$ at room temperature. The cells were viewed using a confocal microscope (FV10i; Olympus Corporation).
Tumor xenograft model. The animal experiments were reviewed and approved by the Animal Care and Experimental Committee of Henan Provincial People's Hospital. Six-week old female BALB $\backslash \mathrm{c}$ nude mice ( $\mathrm{n}=20$; weighing 20 g) were used in the present study. The BABL/c nude mice were housed in individually ventilated cages under specific pathogen-free conditions under 24-h light/dark cycle, $20-26^{\circ} \mathrm{C}$ and $50-80 \%$ humidity. Mice were allowed access to sterilized water and feed ad libitum. The U87 cells $\left(3 \times 10^{6}\right)$ infected with miR-25-3p or miR-ctrl were subcutaneously injected into the nude mice for 2 weeks until visible tumors were observed. The mice were then randomly assigned into the TMZ (TMZ) or vehicle (Veh) group ( $n=10$ for each group). The mice in the TMZ group received daily treatment with $40 \mathrm{mg} / \mathrm{kg}$ TMZ for 3 weeks, while those in the Veh group received an equal volume of PEG-400: PBS (1:1) simultaneously. TMZ was dissolved in PEG-400: PBS (1:1) and administered by oral gavage. Tumor size was evaluated every 3 days using calipers and determined using the following formula: Length $\mathrm{x}$ width $2 / 2$. Finally, the mice were anesthetized by inhalation with $3 \%$ isoflurane and sacrificed by cervical dislocation. The tumors were then dissected and weighed. In the present study, the largest tumor diameter was observed $<2 \mathrm{~cm}$ and the largest tumor volume was $<2,000 \mathrm{~mm}^{3}$.

Luciferase reporter assay. The target of miR-25-3p was predicted using TargetScan 7.2 (http://www.targetscan. org/vert_72/). The 3'UTR of FBXW7 with miR-25-3p binding sites was inserted into the pMIR-REPORT vector (\#AM5795; Thermo Fisher Scientific, Inc.). The QuickChange Site-Directed Mutagenesis kit (\#210518, Agilent Technologies, Inc.) was used to generate mutations in the predicted binding sites for FBXW7 as per the manufacturer's protocol. The 293T cells were co-transfected with miR-25-3p, anti-miR-25-3p or control plasmids, pMIR-REPORT vector containing the 3'UTR of FBXW7 or mutant, and a Renilla luciferase plasmid at a ratio of 2:2:1 using Lipofectamine $3000^{\circledR}$ (Invitrogen; Thermo Fisher Scientific, Inc.) at $37^{\circ} \mathrm{C}$ overnight. Dual-luciferase reporter assay system (Promega Corporation) was performed to determine the luciferase activity at $48 \mathrm{~h}$ post-transfection.

RNA immunoprecipitation (RNA-IP). RNA immunoprecipitation was performed according to the protocol provided with the Magna RIP RNA-Binding Protein Immunoprecipitation kit (EMD Millipore \#17-700). Briefly, cells were washed with ice-cold PBS, then lysed with RIP lysis buffer for $5 \mathrm{~min}$ at $4^{\circ} \mathrm{C}$. Subsequently, $50 \mu 1$ Magnetic Beads Protein A/G (\#CS203178, EMD Millipore) beads were washed with $0.5 \mathrm{ml} \mathrm{RIP}$ wash buffer. The beads $(100 \mu \mathrm{l})$ were then incubated with anti-Agol (5 $\mu \mathrm{g}$, cat. no. ab279392, Abcam) or anti-IgG (5 $\mu \mathrm{g}$; cat. no. ab238004, Abcam) for $30 \mathrm{~min}$ at room temperature. The beads were then washed the with $0.5 \mathrm{ml}$ RIP Wash Buffer. The cell lysates $(100 \mu \mathrm{l})$ were then incubated with antibody-labeled beads in $900 \mu$ l RIP immunoprecipitation buffer at $4^{\circ} \mathrm{C}$ overnight. The beads were then washed with RIP wash buffer for 5 times, and treated with Proteinase K for $30 \mathrm{~min}$ with shaking at $55^{\circ} \mathrm{C}$. Immunoprecipitated RNA in the precipitates was purified using TRIzol ${ }^{\circledR}$ RNA reagent (Invitrogen; Thermo Fisher Scientific, Inc.) and analyzed by RT-qPCR.

miRNA sequencing analysis. miRNA sequencing was carried out as previously described (23). In brief, total RNA was 
extracted using TRIzol ${ }^{\circledR}$ reagent (Invitrogen; Thermo Fisher Scientific, Inc.). Small RNA libraries were prepared according to the NEBNext Small RNA Library Prep Set for Illumina (New England BioLabs) as indicated in the manufacturer's protocol. The libraries were then sequenced on a NextSeq 500 Sequencer (Illumina, Inc.). Small RNA-seq reads were trimmed using Cutadpt (https://cutadapt.readthedocs.io/en/stable/). miRNA expression was quantified using ncPRO-seq (version v1.5.1) (https://sourceforge.net/projects/ncproseq/files/) based on miRbase v19. Differentially expressed miRNAs were defined as those with a $\log _{2}(\mathrm{FC}) \mid>2$ and FDR $<0.01$ change in expression.

Statistical analysis. SPSS 19.0 (SPSS, Inc.) and GraphPad Prism 8.0 (GraphPad Software, Inc.) were used for statistical analysis. A two-tailed unpaired or paired Student's t-test was used to compare differences between 2 groups. One-way ANOVA (with Tukey's post-hoc test) was used to compare differences between multiple groups. The half maximal inhibitory concentration (IC50) of TMZ was evaluated using GraphPad Prism 8.0. Pearson's Chi-squared test was applied to analyze clinical variables. Data are presented as the mean \pm standard deviation (mean $\pm \mathrm{SD}$ ). $\mathrm{P} \leq 0.05$ was considered to indicate a statistically significant difference.

\section{Results}

Exosomes from TMZ-resistant GBM cells promote the proliferation and $T M Z$ resistance of sensitive GBM cells. To examine the exosomes of TMZ-resistant GBM cells, a TMZ-resistant GBM cell line (A172R) was constructed by exposing parental A172 cells to various concentrations of TMZ for 4 months (data not shown). A172 is a frequently used TMZ-sensitive GBM cell line (24). It has been proven that long-term exposure to $\mathrm{TMZ}$ can confer TMZ resistance to A172 cells (24). In the present study, it was found that the A172R cells exhibited increased resistance to TMZ compared with the parental A172 cells, with the TMZ IC50 changing from 114.3 to $1345.8 \mathrm{nM}$ (A172 vs. A172R, Fig. 1A). The resistance of A172R cells to TMZ was also demonstrated by colony formation assay. It was found that TMZ significantly suppressed the colony formation of parental A172 cells, although this effect was diminished in resistant A172R cells (Fig. 1B and C). The exosomes from resistant A172R cells and parental A172 cells were then extracted from the culture medium of these cells. CD81 and CD63 are markers for exosomes (25). It was verified that CD81 and CD63 were expressed only in exosomes extracted from the culture medium of A172R (A172R-exo) and A172 cells (A172-exo), but not in cell lysates (Fig. 1D). To validate the successful internalization of A172R-exo or A172-exo by GBM cells, A172R-exo and A172-exo were labeled with the green fluorescent dye, PKH67, then added into the culture medium of A172 cells. It was found that the A172 cells treated with A172R-exo or A172-exo exhibited green fluorescent signals; however, the A172 cells treated with PBS did not exhibit any fluorescent signals, suggesting that A172R-exo and A172-exo were successfully internalized by the GBM cells (Fig. 1E and F). Subsequently, the effects of exosomes of A172R and A172 cells on the GBM cells were examined by cell viability assay and soft agar assay. U87 is a frequently used TMZ-sensitive GBM cell line (24). It was found that exosomes of A172R cells (A172R-exo) evidently facilitated the growth of U87 and A172 cells compared with exosomes derived from A172 cells (A172-exo) (Fig. 1G). In addition, the U87 and A172 cells treated with A172R-exo formed larger colonies in soft agar compared with the cells treated with A172-exo (Fig. 1H and I). The effects of A172R-exo and A172-exo on the resistance of GBM cells to TMZ were then evaluated. It was found that the U87 and A172 cells exposed to A172R-exo exhibited an increased TMZ IC50 compared with those treated with PBS or A172-exo, indicating that the exosomes of TMZ-resistant A172R cells spread TMZ resistance to sensitive GBM cells (Fig. 1J). In colony formation assay, the U87 and A172 cells co-treated with TMZ and A172R-exo formed more colonies than the cells co-treated with TMZ and PBS or A172-exo (Fig. 1K and L). Moreover, the U87 and A172 cells co-treated with TMZ and A172R-exo (A172R-exo + TMZ) exhibited a decreased apoptosis compared with the cells co-treated with TMZ and A172-exo (A172-exo + TMZ) (Fig. 1M and N). Western blot analysis revealed that the U87 and A172 cells co-treated with TMZ and A172R-exo (A172R-exo + TMZ) exhibited low levels of cleaved PARP and cleaved caspase-3 compared with the cells co-treated with TMZ and A172-exo (A172-exo + TMZ) (Fig. 10 and P). Taken together, these data demonstrated that the exosomes of TMZ-resistant GBM cells promoted the proliferation and TMZ resistance of sensitive GBM cells.

miR-25-3p is upregulated in exosomes of TMZ-resistant GBM cells and in serum of patients with GBM treated with $T M Z$. Exosomes are enriched with miRNAs (8). Previous studies have indicated that exosomal miRNAs are crucial for acquired TMZ resistance in GBM $(11,14,15)$. In the present study, to explore the possible exosomal miRNAs involved in the TMZ resistance of A172R cells, miRNA sequencing data for A172R-exo and A172-exo were obtained using Illumina NextSeq 500 sequencing. The dysregulated miRNAs between A172R-exo and A172-exo were defined as llog2 fold changel $\geq 2$. The top 20 upregulated miRNAs in A172R-exo compared with A172-exo are depicted in Fig. 2A. To search for exosomal miRNAs that involved in the effects of A172R-exo, A172 cells were treated with A172R-exo in combination with antagomirs for indicated miRNAs, and cell viability was then evaluated. As shown in Fig. 2B, it was found that the depletion of exosomal miR-25-3p significantly impaired the effects of A172R-exo on the viability of A172 cells. Moreover, previous studies have indicated that miR-25-3p plays a key role in the tumorigenesis of GBM $(26,27)$. In the present study, miR-25-3p expression in A172R cells and exosomes was determined by RT-qPCR. It was found that miR-25-3p expression was upregulated in A172R cells, as well as exosomes derived from these cells (A72R-exo) (Fig. 2C and D). As miR-25-3p was overexpressed in A172R-exo, it was hypothesized that miR-25-3p expression may be increased in serum from patients with GBM treated with TMZ. Thus, circulating miR-25-3p in healthy donors and patients with GBM treated with or without TMZ was assayed by RT-qPCR. As was expected, miR-25-3p expression was upregulated in patients with GBM treated with TMZ (GBM-TMZ) compared with untreated patients with GBM or healthy donors (Fig. 2E). Furthermore, the association of circulating miR-25-3p with the clinicopathological characteristics of patients with GBM was evaluated. Patients with GBM were divided into the serum 
A

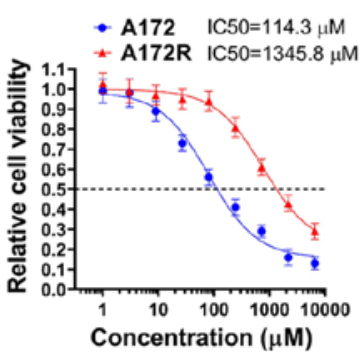

E

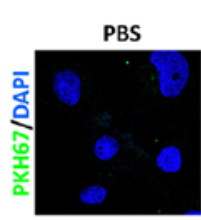

B

A172

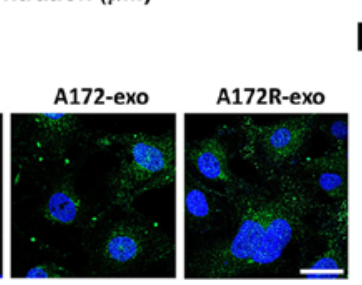

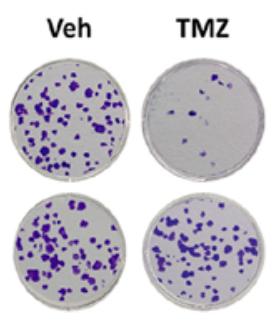

$\mathbf{F}$

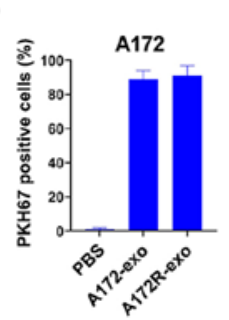

H
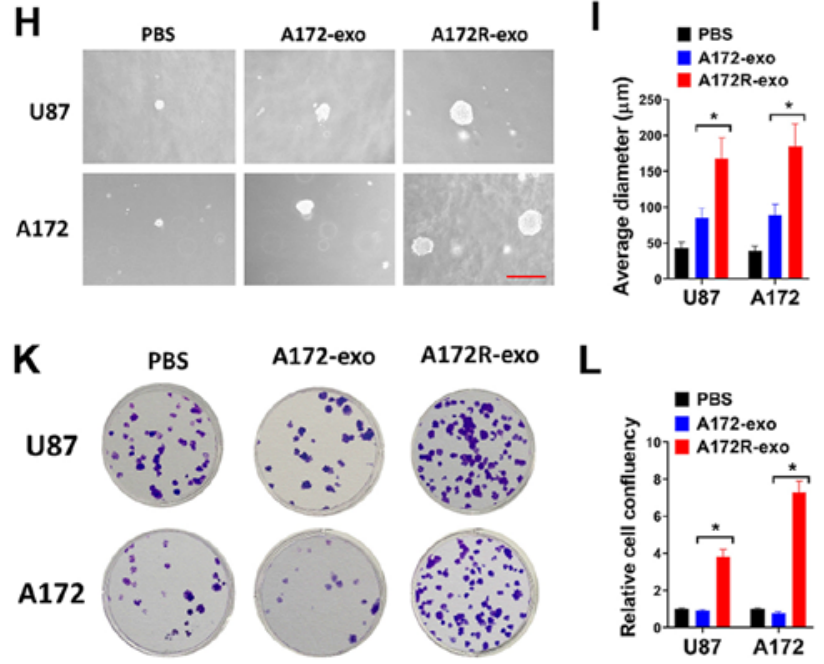

A172R-exo

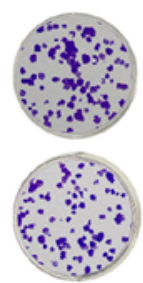

L
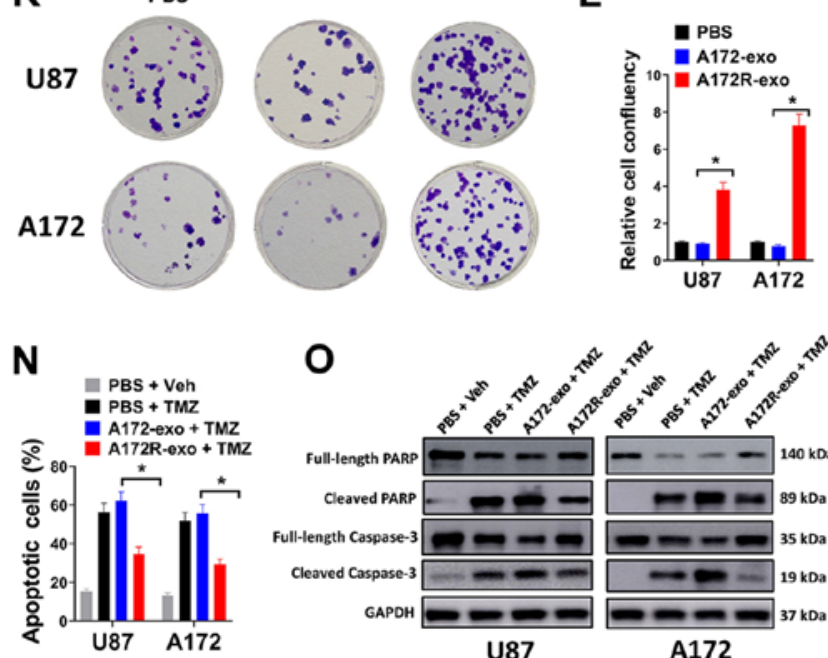

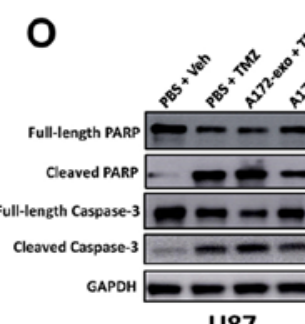

U87

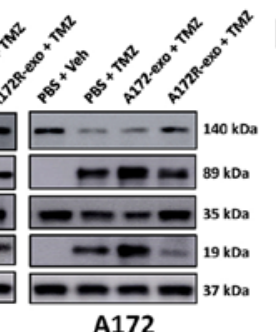

C

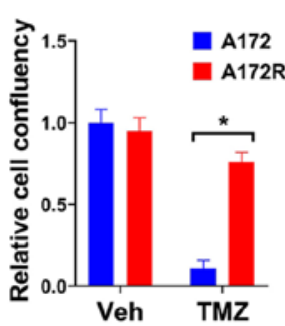

G

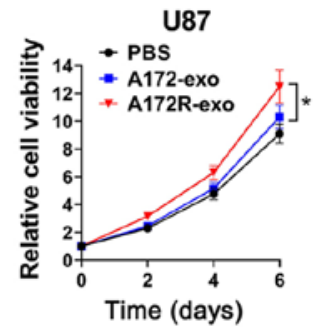

U87

J

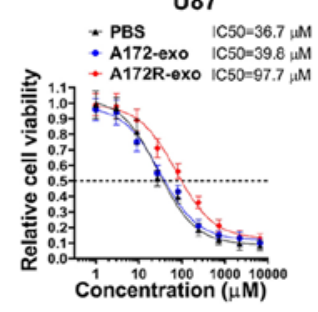

D
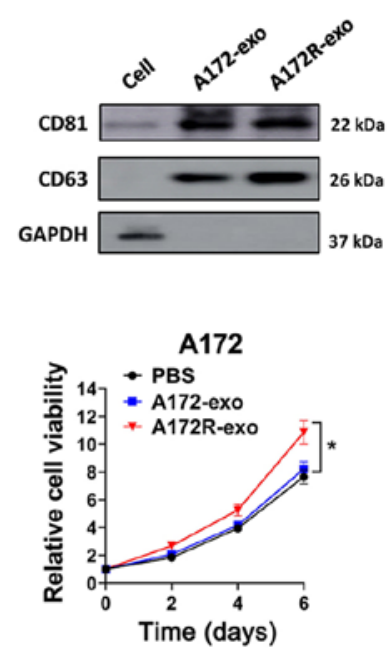

A172

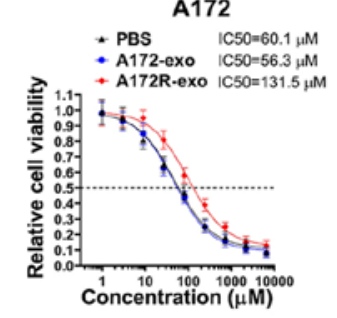

M
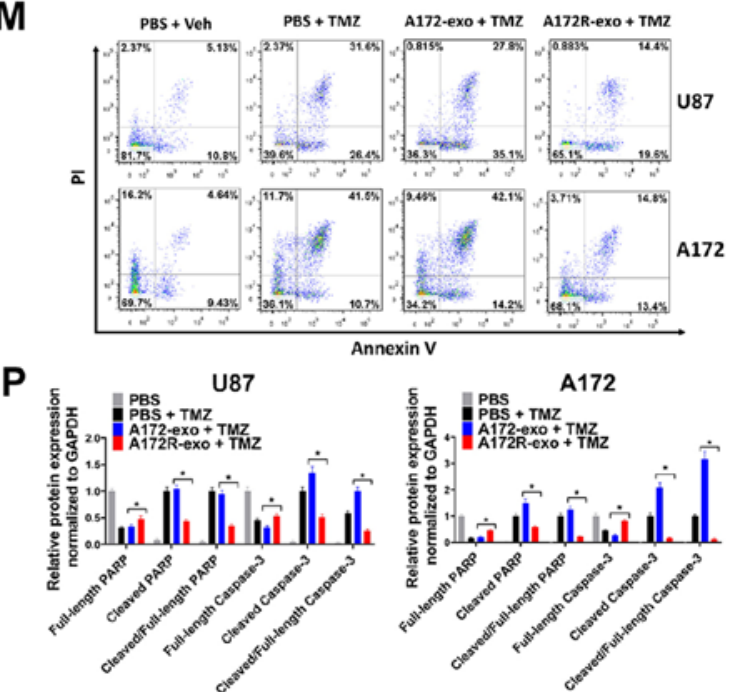

Figure 1. Exosomes from TMZ-resistant GBM cells promote the proliferation and TMZ resistance of sensitive GBM cells. (A) A172R or A172 cells were seeded in 96 -well plates $(5,000$ cells/well), then exposed to $0,1,3,9,27,81,243,729,2,181$ or $6,543 \mu \mathrm{M}$ TMZ for 6 days, then evaluated by cell viability assay. (B and C) A172R or A172 cells were seeded in 6-well plates (3,000 cells/well) and exposed to $50 \mu \mathrm{M}$ TMZ (TMZ group) or an equal volume of DMSO (Veh group) for colony formation assay. (B) Representative plates and (C) relative cell confluency are shown. (D) Expression of the exosomal markers, CD81 and CD63, was evaluated by western blot analysis. (E) A172 cells were incubated with PKH67-labeled A172-exo or A172R-exo, or PBS for exosome internalization assay. DAPI was used to stain the nuclei. Scale bar, $10 \mu \mathrm{m}$. (F) The percentages of PKH67-positive cells are shown. (G) U87 or A172 cells were treated with A172R-exo $(50 \mu \mathrm{g} / \mathrm{ml})$, A172-exo $(50 \mu \mathrm{g} / \mathrm{ml})$ or an equal volume of PBS, and cell viability was evaluated on days 2, 4 and 6 . (H and I) U87 or A172 cells were seeded at 8,000 cells/well for soft agar assay, and simultaneously treated with A172R-exo $(50 \mu \mathrm{g} / \mathrm{ml})$, A172-exo $(50 \mu \mathrm{g} / \mathrm{ml})$ or an equal volume of PBS. Represent images of $(\mathrm{H})$ colonies (scale bar, $200 \mu \mathrm{m}$ ) and (I) the average diameter of colonies are shown. (J) U87 or A172 cells were seeded in 96-well plates (5,000 cells/well), then exposed to $0,1,3,9,27,81,243,729,2,181$ or $6,543 \mu \mathrm{M} \mathrm{TMZ}$ in combination with A172R-exo (50 $\mu \mathrm{g} / \mathrm{ml})$, A172-exo (50 $\mu \mathrm{g} / \mathrm{ml})$ or an equal volume of PBS for 6 days, then evaluated by cell viability assay. (K and L) U87 or A172 cells were seeded in 6 -well plates (3,000 cells/well) and treated with $50 \mu \mathrm{M}$ TMZ in combination with A172R-exo $(50 \mu \mathrm{g} / \mathrm{ml})$, A172-exo $(50 \mu \mathrm{g} / \mathrm{ml})$ or an equal volume of PBS for colony formation assay. Represent (K) plates and (L) relative cell confluency are shown. (M-P) U87 or A172 cells were treated with $50 \mu \mathrm{M} \mathrm{TMZ}$ or an equal volume of DMSO (Veh) in combination with A172R-exo (50 $\mu \mathrm{g} / \mathrm{ml}$ ), A172-exo $(50 \mu \mathrm{g} / \mathrm{ml})$ or an equal volume of PBS for $72 \mathrm{~h}$, then cells were used for (M) flow cytometric analysis or $(\mathrm{O})$ western blot analysis. (N) Percentages of apoptotic cells and (P) relative protein expression normalized to GAPDH are shown. All experiments were performed in triplicate. "P $\leq 0.05$. TMZ, temozolomide; GBM, glioblastoma; A172R, TMZ-resistant A172 cells; A172-exo or A172R-exo, exosomes from A172 and A172R cells, respectively.

miR-25-3p high expression group and the serum miR-25-3p low expression group using the median of miR-25-3p expression as the cut-off value. It was found that a high circulating miR-25-3p level was positively associated with a larger tumor size and 
A

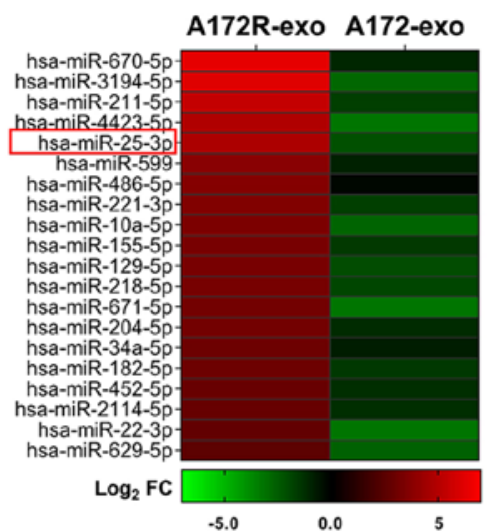

B

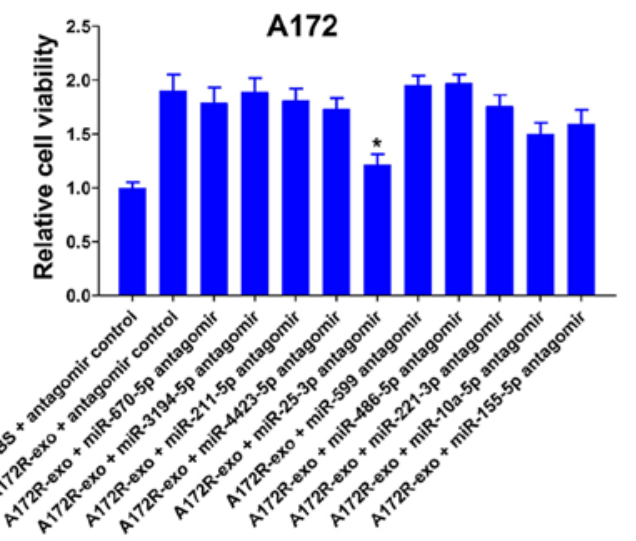

C

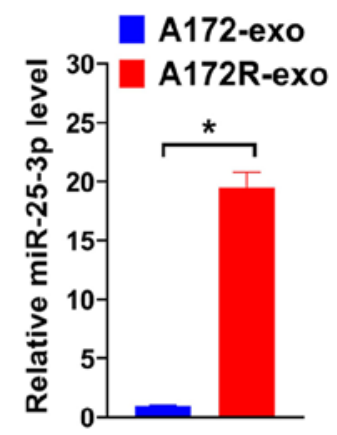

D

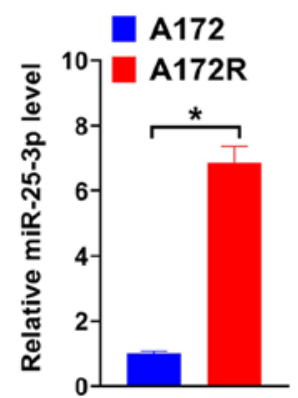

E

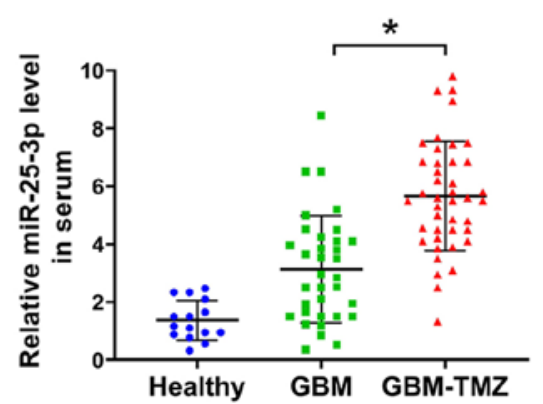

Figure 2. miR-25-3p is upregulated in exosomes of TMZ-resistant GBM cells and in serum of patients with GBM treated with TMZ. (A) Heatmap illustrating the top 20 upregulated miRNAs in exosomes derived from A172R-exo compared with exosomes derived from parental A172-exo. (B) A172 cells were treated with A172R-exo $(50 \mu \mathrm{g} / \mathrm{ml})$ or an equal volume of PBS in combination with antagomir for the indicated miRNAs for 6 days, and cell viability was then evaluated. (C and D) Relative miR-25-3p expression in (C) exosomes or (D) cell lines was evaluated by RT-qPCR. (E) Relative expression of miR-25-3p in serum of patients with GBM treated with TMZ, untreated GBM patients, or healthy donors was evaluated by RT-qPCR. All experiments were performed in triplicate. "P $\leq 0.05$. TMZ, temozolomide; GBM, glioblastoma; A172R, TMZ-resistant A172 cells; A172-exo or A172R-exo, exosomes from A172 and A172R cells, respectively.

TMZ resistance in patients with GBM (Table I). On the whole, these data suggested that miR-25-3p expression was increased in exosomes from TMZ-resistant GBM cells and in serum of patients with GBM treated with TMZ.

Knockdown of exosomal miR-25-3p partially abrogates the effects induced by exsomes transferred from TMZ-resistant GBM cells. To explore the possible role of exosomal miR-25-3p in TMZ-resistant GBM cells, miR-25-3p expression was depleted by transduction with anti-miR-25-3p. It was found that the transduction of anti-miR-25-3p significantly decreased the expression levels of miR-25-3p in the A172R, A172 and U87 cells (Fig. 3A). Moreover, exosomal miR-25-3p expression was significantly downregulated in the A172R cells transfected with anti-miR-25-3p (A172R-exo + anti-miR-25-3p group) compared with the cells transfected with anti-miR-ctrl (A172R-exo + anti-miR-ctrl group) (Fig. 3B). In the cell viability assay (Fig. 3C) and soft agar assay (Fig. 3D and E), it was observed that the depletion of miR-25-3p attenuated the effects of A172R-exo on the growth and colony formation of U87 and A172R cells. Moreover, the U87 and A172 cells treated with A172R-exo exhibited an increased TMZ IC50 compared with the cells treated with PBS; however, these effects were attenuated by miR-25-3p knockdown (Fig. 3F). In the colony formation assay, the U87 and A172 cells treated with A172R-exo (A172R-exo + anti-miR-ctrl) exhibited increased colony formation compared with the cells treated with PBS; however, these effects were partially attenuated by miR-25-3p knockdown
(A172R-exo + anti-miR-25-3p) (Fig. 3G and H). In flow cytometric analysis, the U87 and A172 cells co-treated with TMZ and A172R-exo (A172R-exo + anti-miR-ctrl + TMZ) exhibited a decreased apoptosis compared with the cells co-treated with PBS and TMZ (PBS + TMZ); however, these effects were partially attenuated by miR-25-3p knockdown (A172R-exo + anti-miR-25-3p + TMZ) (Fig. 3I and J). In western blot analysis, the U87 and A172 cells co-treated with TMZ and A172R-exo (A172R-exo + anti-miR-ctrl + TMZ) exhibited low levels of cleaved PARP and cleaved caspase-3 compared with the cells co-treated with PBS and TMZ (PBS + TMZ); however, these effects were diminished by miR-25-3p knockdown (A172R-exo + anti-miR-25-3p + TMZ) (Fig. 3K and L). Collectively, these data indicated that the knockdown of exosomal miR-25-3p partially abrogated the effects induced by exsomes transferred from TMZ-resistant GBM cells.

miR-25-3p overexpression promotes the proliferation and $T M Z$ resistance of GBM cells. The effects of miR-25-3p overexpression on GBM cells were evaluated in the present study. miR-25-3p overexpression was achieved by transfecting the cells with lentivirus expressing miR-25-3p (Fig. 4A). It was found that the enforced expression of $\mathrm{miR}-25-3 \mathrm{p}$ promoted the growth of U87 and A172 cells compared with the cells transfected with miR-ctrl (Fig. 4B). In BrdU incorporation assay (Fig. 4C and D) and soft agar assay (Fig. 4E and F), miR-25-3p overexpression increased the number of BrdU-positive cells and the diameter of the colonies of U87 and A172 cells. These 
A

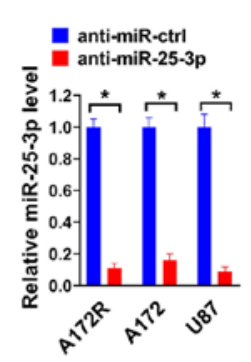

B

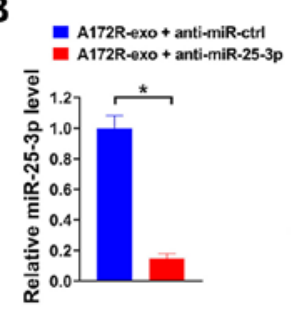

C

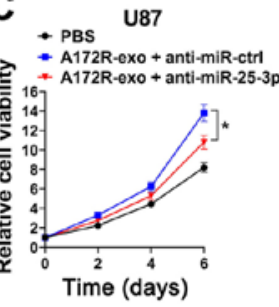

A172

- PBS

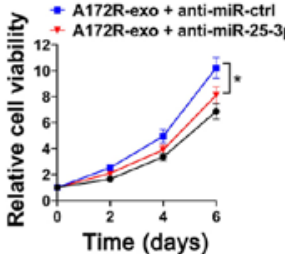

D

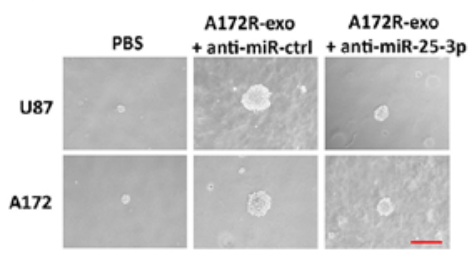

E - $P B S$

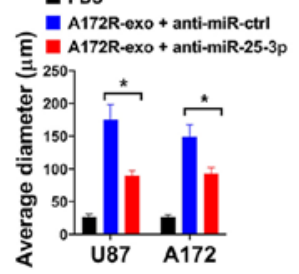

$\mathbf{F}$

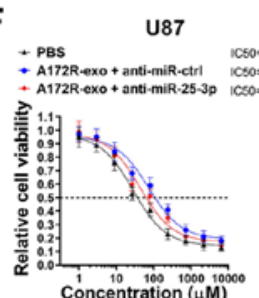

A172
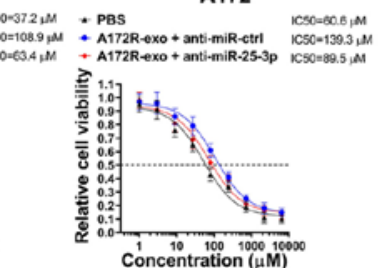
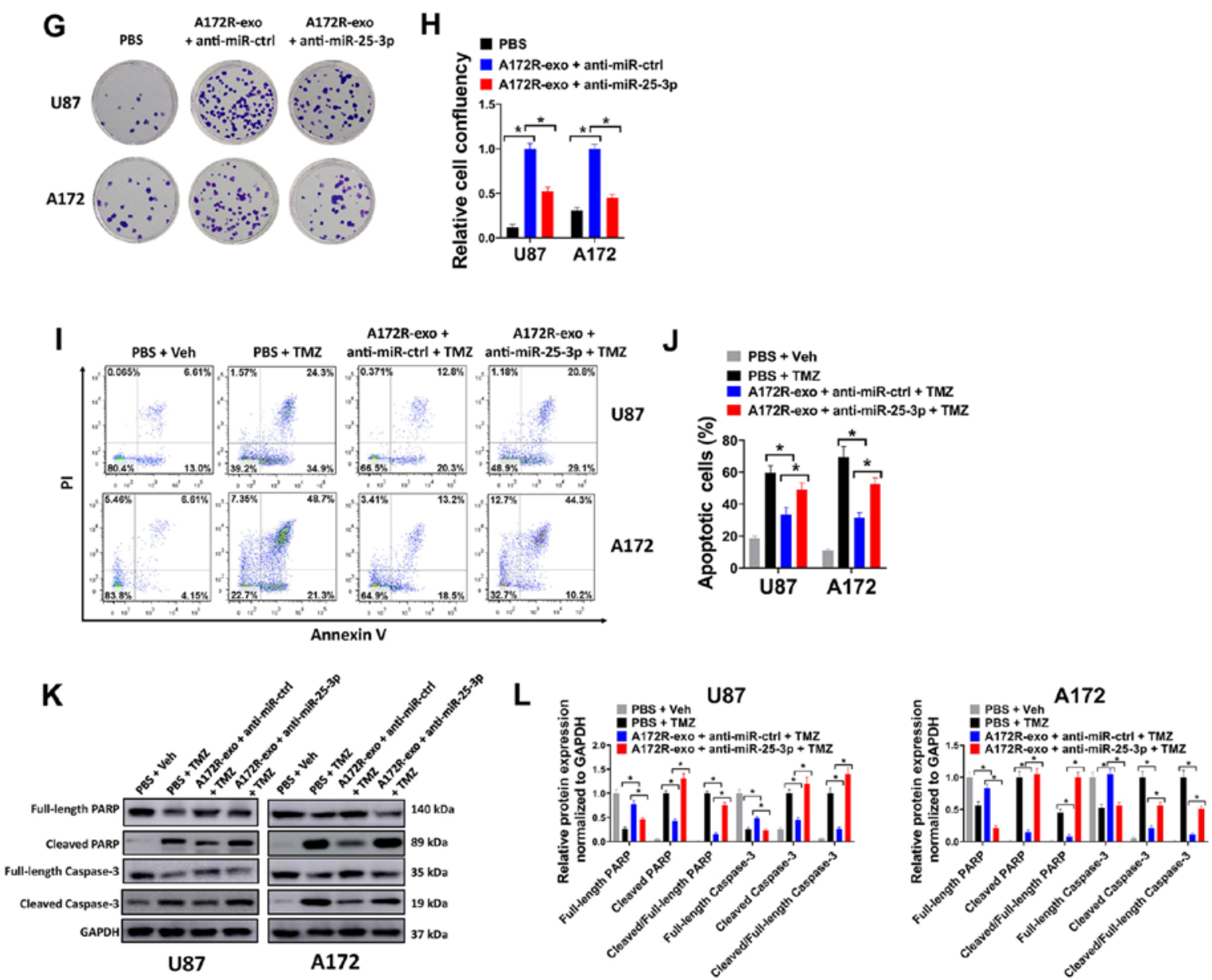

Figure 3. Knockdown of exosomal miR-25-3p partially abrogates the effects caused by exsomes transferred from TMZ-resistant GBM cells. (A) Relative miR-25-3p expression in A172R, A172 and U87 cells transduced with anti-miR-25-3p or anti-miR-ctrl was evaluated by RT-qPCR. (B) Relative miR-25-3p expression in exosomes derived from A172R cells transduced with anti-miR-25-3p (A172R-exo + anti-miR-25-3p group) or exosomes derived from A172R cells transduced with anti-miR-ctrl (A172R-exo + anti-miR-ctrl group) was evaluated by RT-qPCR. (C) U87 or A172 cells were treated with exosomes (50 $\mu \mathrm{g} / \mathrm{ml})$ derived from A172R cells transduced with anti-miR-25-3p or miR-ctrl, or PBS, and cell viability was then evaluated on days 2, 4 and 6. (D and E) U87 or A172 cells were seeded at 8,000 cells/well for soft agar assay, and simultaneously treated with exosomes $(50 \mu \mathrm{g} / \mathrm{ml})$ derived from A172R cells transduced with anti-miR-25-3p or miR-ctrl, or PBS. (D) Representative images of colonies (scale bar, $200 \mu \mathrm{m}$ ) and (E) average diameter of colonies are shown. (F) U87 or A172 cells were seeded in 96-well plates $(5,000$ cells/well), then exposed to $0,1,3,9,27,81,243,729,2,181$ or $6,543 \mu \mathrm{M}$ TMZ in combination with exosomes $(50 \mu \mathrm{g} / \mathrm{ml})$ derived from A172R cells transduced with anti-miR-25-3p or miR-ctrl, or PBS for 6 days, and cell viability assay was then performed. $(\mathrm{G}$ and $\mathrm{H}) \mathrm{U} 87$ or A172 cells were seeded in 6-well plates (3,000 cells/well) and treated with $50 \mu \mathrm{M} \mathrm{TMZ}$ in combination with exosomes $(50 \mu \mathrm{g} / \mathrm{ml}) \mathrm{derived}$ from A172R cells transduced with anti-miR-25-3p or miR-ctrl, or PBS for colony formation assay. (G) Represent plates and (H) relative cell confluency are shown. (I-L) U87 or A172 cells were treated with $50 \mu \mathrm{M} \mathrm{TMZ}$ or an equal volume of DMSO (Veh) in combination with exosomes (50 $\mu \mathrm{g} / \mathrm{ml}) \mathrm{derived}$ from A172R cells transduced with anti-miR-25-3p or miR-ctrl, or PBS for $72 \mathrm{~h}$, then cells were used for (I) flow cytometry or (K) western blot analysis. (J) Percentages of apoptotic cells and (L) relative protein expression normalized to GAPDH are shown. All experiments were performed in triplicate. ${ }^{*} \mathrm{P} \leq 0.05$. TMZ, temozolomide; GBM, glioblastoma; A172R, TMZ-resistant A172 cells; A172-exo or A172R-exo, exosomes from A172 and A172R cells, respectively. 

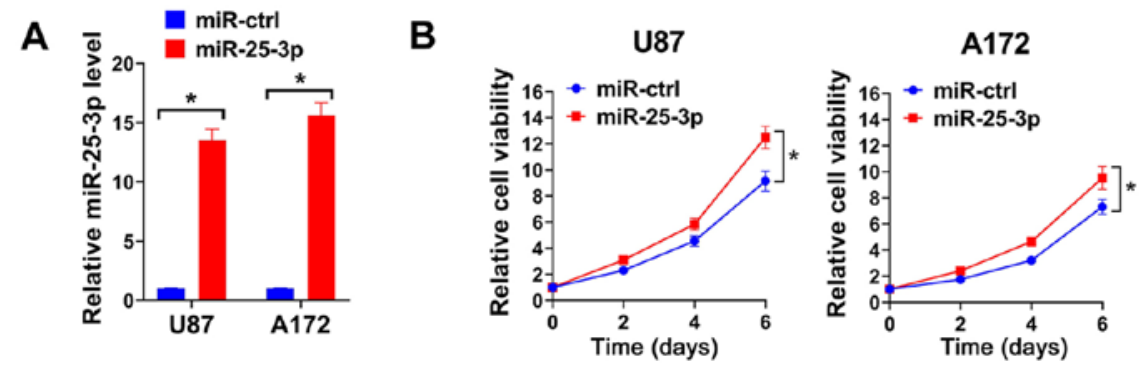
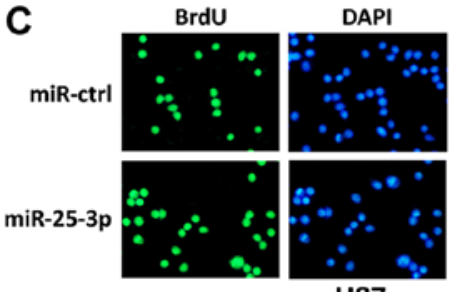

U87

$\mathbf{E}$
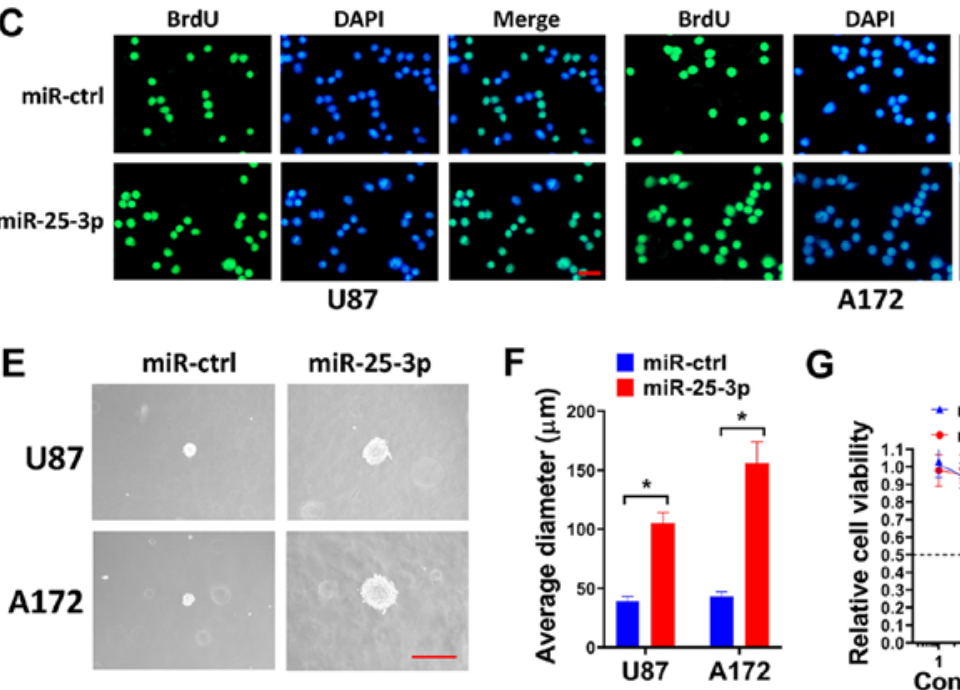

A172

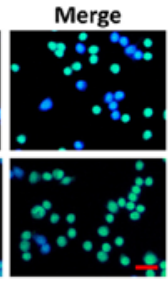

G
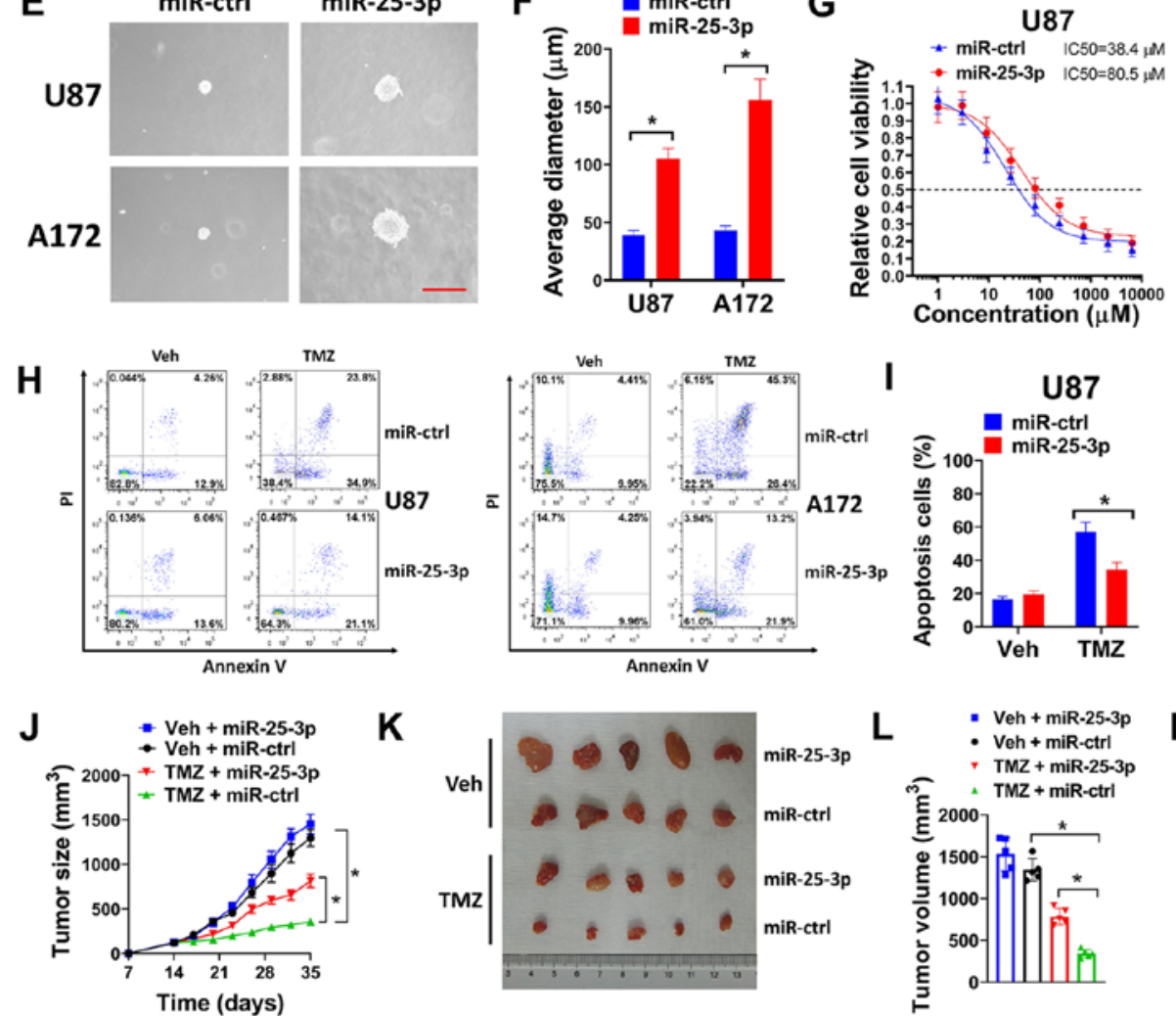
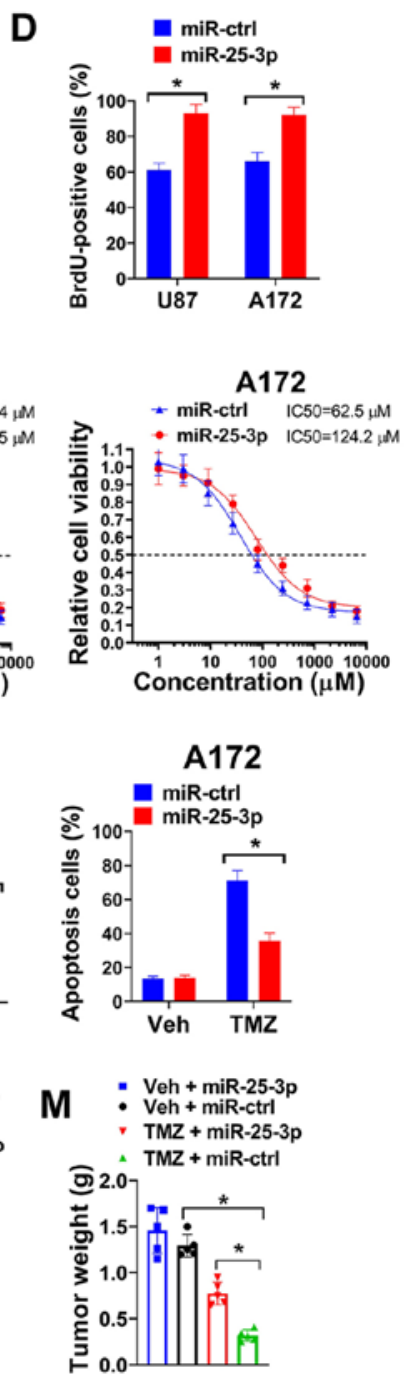

Figure 4. miR-25-3p overexpression promotes the proliferation and TMZ resistance of GBM cells. (A) U87 or A172 cells were transduced with miR-25-3p expression lentivirus or miR-ctrl, and the relative miR-25-3p expression was then evaluated by RT-qPCR. (B) U87 or A172 cells transduced with miR-25-3p or miR-ctrl were seeded in 96-well plates (5,000 cells/well), and cell viability was then evaluated on days 2, 4 and 6 . (C and D) U87 or A172 cells were transduced with miR-25-3p expression lentivirus or miR-ctrl, then seeded on coverslips for BrdU incorporation assay. (C) Represent images (scale bar, $50 \mu \mathrm{m}$ ) and (D) percentages of BrdU-positive cells are shown. (E and F) U87 or A172 cells transduced with miR-25-3p expression lentivirus or miR-ctrl were seeded in 6-well plates (8,000 cells/well) for soft agar assay. (E) Represent images of colonies (scale bar, $200 \mu \mathrm{m})$ and (F) the average diameter of colonies are shown. (G) U87 or A172 cells transduced with miR-25-3p expression lentivirus or miR-ctrl were seeded in 96-well plates (5,000 cells/well), then exposed to $0,1,3,9,27,81,243,729,2,181$ or $6,543 \mu \mathrm{M}$ TMZ for 6 days and cell viability assay was then performed. (H and I) U87 or A172 cells transduced with miR-25-3p expression lentivirus or miR-ctrl were treated with $50 \mu \mathrm{M}$ TMZ for $72 \mathrm{~h}$, and (H) the cells were then used for flow cytometry. (I) Percentages of apoptotic cells are shown. (J-M) U87 cells $\left(3 \times 10^{6}\right)$ transduced with miR-25-3p or miR-ctrl were subcutaneously injected into nude mice for 2 weeks, and the mice were then treated with $40 \mathrm{mg} / \mathrm{kg}$ TMZ (TMZ group) or an equal volume of PEG-400: PBS (1:1) (Veh group) daily for 3 weeks. (J) Tumor growth, $(\mathrm{K})$ representative images of tumors, $(\mathrm{L})$ tumor volume and $(\mathrm{M})$ tumor weight are shown for each group. All experiments were performed in triplicate. $\mathrm{P} \leq 0.05$. TMZ, temozolomide; GBM, glioblastoma.

data suggested that miR-25-3p overexpression increased the proliferation of GBM cells. The effects of miR-25-3p on the TMZ resistance of GBM cells were then evaluated. In cell viability assay, miR-25-3p overexpression markedly increased the TMZ IC50 of U87 and A172 cells compared with the miR-ctrl (Fig. 4G). Flow cytometric analysis indicated that miR-25-3p overexpression decreased the number of apoptotic cells treated with TMZ (Fig. $4 \mathrm{H}$ and I). In the tumor xenograft 
A

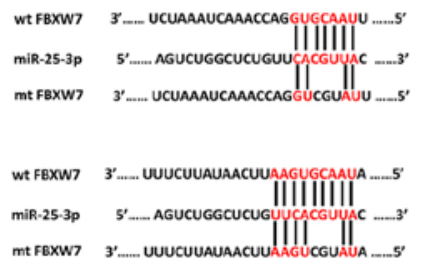

B

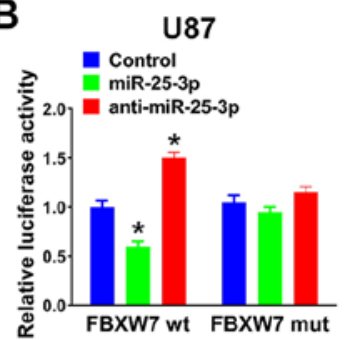

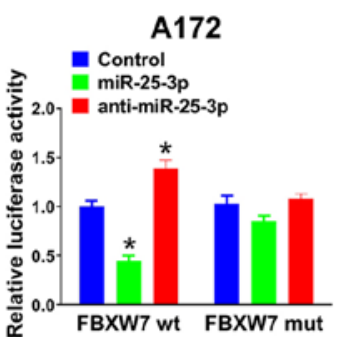

$\mathbf{F}$
C U87

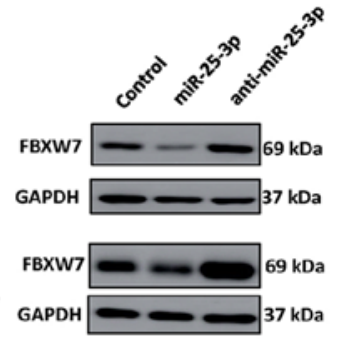

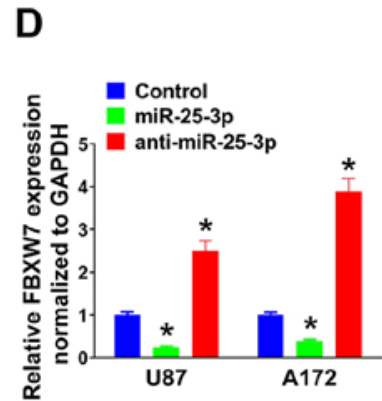

G

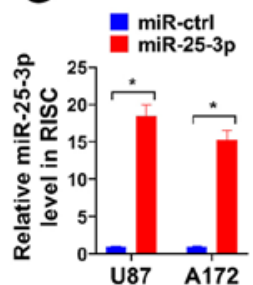

E

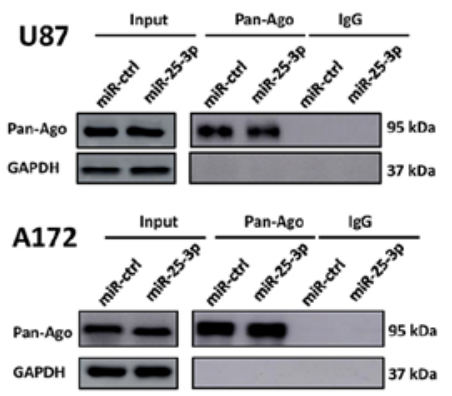

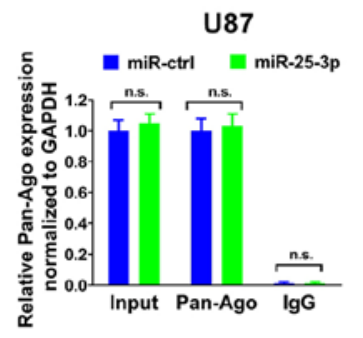

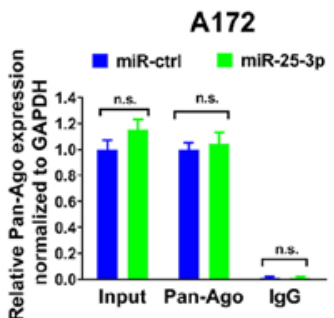

$\mathbf{J}$

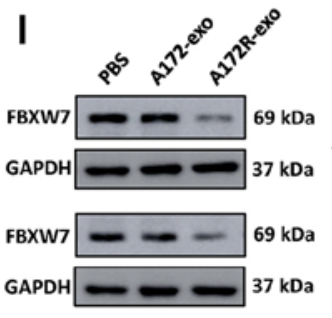

H

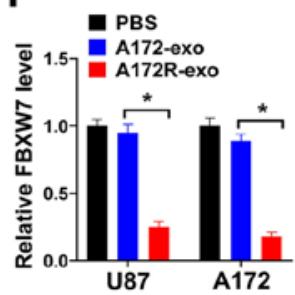

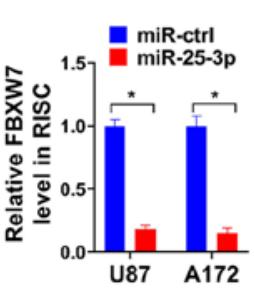

Figure 5. FBXW7 is a direct target of miR-25-3p in GBM cells. (A) Binding sites for miR-25-3p and FBXW7 were predicted using TargetScan 7.2. A 3-bp mutation at the binding sites of miR-25-3p and FBXW7 was generated at the 3'UTR of FBXW7 using a QuickChange site-directed mutagenesis kit. (B) 293T cells co-transfected with miR-25-3p, anti-miR-25-3p or control plasmids, pMIR-REPORT vector containing the 3'UTR of FBXW7 or mutant, or a Renilla luciferase plasmid for luciferase reporter assay. (C and D) U87 or A172 cells were transduced with miR-25-3p, anti-miR-25-3p or control plasmids, and the protein expression of FBXW7 was then evaluated by western blot analysis. (D) Relative protein expression levels normalized to GAPDH are shown. (E-G) U87 or A172 cells transduced with miR-25-3p expression lentivirus or miR-ctrl were used for RNA-IP analysis. (E and F) Protein expression of Pan-Ago was evaluated by western blot analysis. The antibodies of anti-Agol and anti-IgG cannot pull down GAPDH; thus, no bands are shown here. (F) Relative protein levels normalized to GAPDH are shown. (G) Relative expression of miR-25-3p or FBXW7 was evaluated by RT-qPCR. (H-J) U87 or A172 cells were treated with A172R-exo $(50 \mu \mathrm{g} / \mathrm{ml})$, A172-exo $(50 \mu \mathrm{g} / \mathrm{ml})$ or an equal volume of PBS, and the relative (H) mRNA or (I-J) protein expression of FBXW7 was evaluated by RT-qPCR or western blot analysis, respectively. ( $\mathrm{J})$ Relative protein expression levels normalized to GAPDH are shown. All experiments were performed in triplicate. ${ }^{*} \mathrm{P} \leq 0.05$. FBXW7, F-box and WD repeat domain containing 7; GBM, glioblastoma.

model, U87 cells infected with miR-25-3p or miR-ctrl were subcutaneously injected into nude mice and the mice were then treated with TMZ or the vehicle control for 3 weeks. It was found that TMZ treatment significantly reduced the tumor growth of U87 cells transduced with miR-ctrl (TMZ + miR-ctrl group) compared with the Veh + miR-ctrl group, with reduced tumor volume and weight (Fig. 4J-M). However, the U87 cells transduced with miR-25-3p (TMZ + miR-25-3p group) led to increased tumor growth, volume and weight compared with the cells transduced with miR-ctrl when the mice were treated with TMZ (TMZ + miR-ctrl group) (Fig. 4J-M). Taken together, these findings demonstrated that miR-25-3p overexpression promoted the proliferation and TMZ resistance of GBM cells.

FBXW7 is a direct target of miR-25-3p in GBM cells. To clarify the underlying mechanisms of miR-25-3p in GBM, the target of miR-25-3p was predicted using TargetScan 7.2. FBXW7 was predicted to interact with miR-25-3p in the present study (Fig. 5A). FBXW7 is a well-characterized tumor suppressor with a role in tumorigenesis and the TMZ tolerance of GBM $(28,29)$. In the present study, the interaction between miR-25-3p and FBXW7 was validated by luciferase reporter assay. A 3-bp mutation at the binding sites of miR-25-3p and FBXW7 was generated at the 3'UTR of FBXW7 (mt FBXW7) (Fig. 5A). It was found that miR-25-3p overexpression markedly inhibited the luciferase activity of the 3'UTR of wt FBXW7, while miR-25-3p knockdown evidently increased the luciferase activity of the 3'UTR of wt FBXW7 in U87 and A172 cells (Fig. 5B). Furthermore, a 3-bp mutation at the binding sites of miR-25-3p and FBXW7 abolished the effects induced by miR-25-3p overexpression or knockdown (Fig. 5B). Furthermore, it was found that miR-25-3p overexpression decreased FBXW7 expression in the U87 and A172 cells, while miR-25-3p knockdown exerted opposite effects (Fig. 5C and D). Subsequently, RNA-IP assay was performed to identify mRNA levels in the Ago/ RNA-induced silencing complex (RISC) following miR-25-3p overexpression (Fig. 5E-G). Firstly, it was found that miR-25-3p overexpression had no effect on the protein expression of Pan-Ago in the U87 and A172 cells (Fig. 5E and F). It was then proven that miR-25-3p overexpression evidently increased the levels 

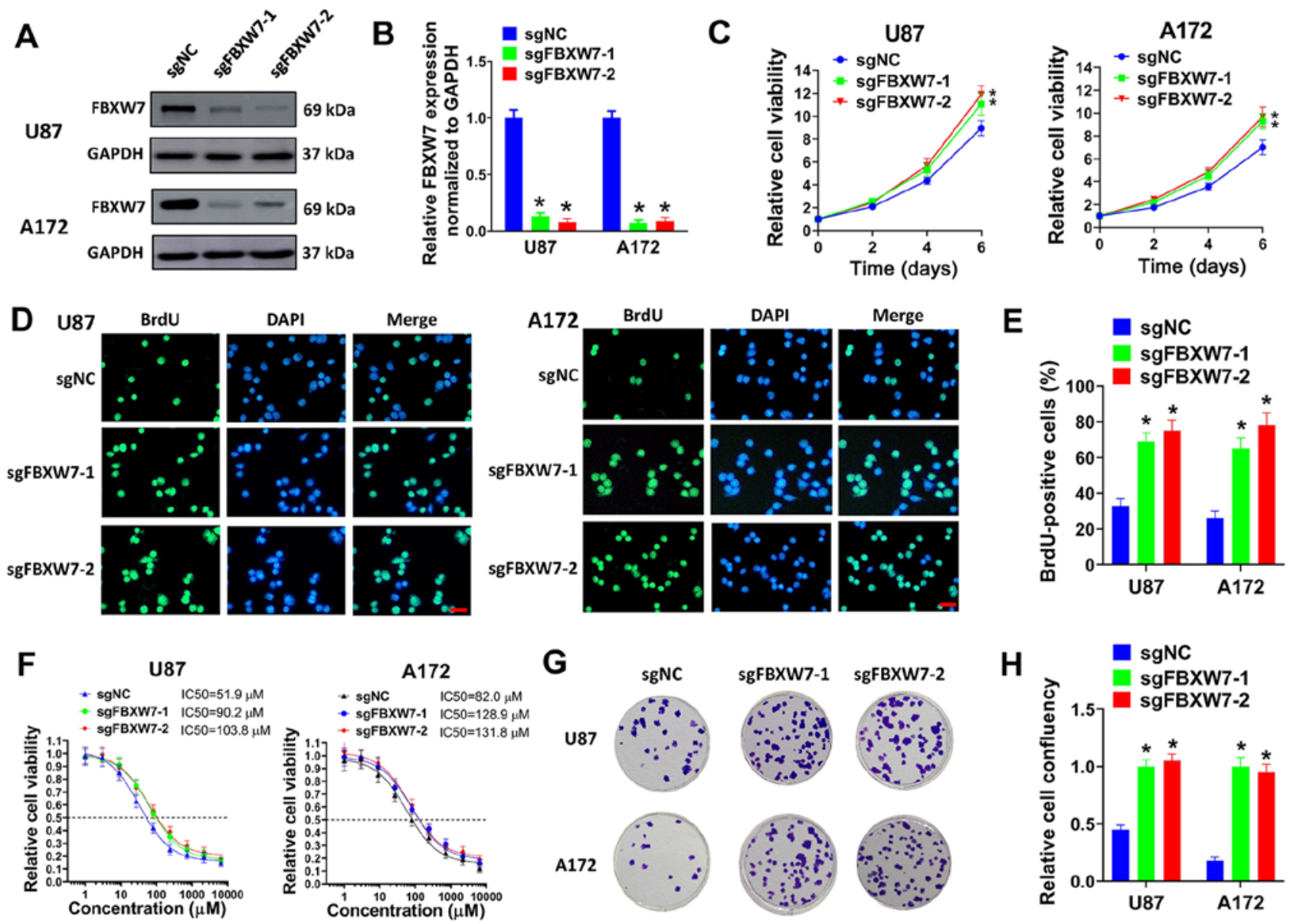

Figure 6. FBXW7 knockdown promotes the proliferation and TMZ resistance of GBM cells. (A and B) U87 or A172 cells were transduced with sgFBXW7-1, sgFBXW7-2 or sgNC, and (A) the protein expression of FBXW7 was evaluated by western blot analysis. (B) Relative protein levels normalized to GAPDH are shown. (C) U87 or A172 cells transduced with sgFBXW7-1, sgFBXW7-2 or sgNC were seeded in 96-well plates (5,000 cells/well), and cell viability was then evaluated on days 2, 4 and 6. (D and E) U87 or A172 cells were transduced with sgFBXW7-1, sgFBXW7-2 or sgNC, and then seeded on coverslips for BrdU incorporation assay. (D) Represent images (scale bar, $50 \mu \mathrm{m}$ ) and (E) percentages of BrdU-positive cells are shown. (F) U87 or A172 cells transduced with sgFBXW7-1, sgFBXW7-2 or sgNC were seeded in 96-well plates (5,000 cells/well), then exposed to $0,1,3,9,27,81,243,729,2,181$ or $6,543 \mu \mathrm{M}$ TMZ for 6 days and evaluated by cell viability assay. (G and H) U87 or A172 cells transduced with sgFBXW7-1, sgFBXW7-2 or sgNC were seeded in 6-well plates (3,000 cells/well) and treated with $50 \mu \mathrm{M} \mathrm{TMZ}$ for colony formation assay. (G) Represent plates and $(\mathrm{H})$ relative cell confluency are shown. All experiments were performed in triplicate. ${ }^{*} \mathrm{P} \leq 0.05$. FBXW7, F-box and WD repeat domain containing 7; GBM, glioblastoma.

of miR-25-3p incorporated into the RISC (Fig. 5G, left panel). Moreover, the levels of FBXW7 incorporated into the RISC were markedly reduced by the enforced expression of miR-25-3p (Fig. 5G, right panel). As miR-25-3p expression was upregulated in exosomes derived from A72R cells (A172R-exo), the FBXW7 level we also evaluated in U87 and A172 cells treated with A172R-exo or A172-exo. It was proven that the mRNA and protein levels of FBXW7 were markedly decreased by treatment with A172R-exo (Fig. 5H-J). These data demonstrated that FBXW7 was targeted by miR-25-3p in GBM cells.

FBXW7 knockdown promotes the proliferation and TMZ resistance of GBM cells. To clarify the function of FBXW7 in GBM, FBXW7 was knocked down in GBM cells and cell viability, BrdU incorporation and colony formation assays were then performed. Two sgRNAs of FBXW7 (sgFBXW7-1 and sgFBXW7-2) were constructed and introduced into the U87 and A172 cells. It was found that FBXW7 was successfully knock down by these two sgRNAs (Fig. 6A and B). In cell viability assay, FBXW7 knockdown promoted the growth of U87 and A172 cells (Fig. 6C). In BrdU incorporation assay, the knockdown of FBXW7 evidently increased the percentage of BrdU-positive cells (Fig. 6D and E). Moreover, it was found that FBXW7 knockdown increased the TMZ IC50 in U87 and A172 cells (Fig. 6F). In colony formation assay, FBXW7 knockdown facilitated the colony formation of U87 and A172 cells co-treated with TMZ (Fig. 6G and H). Collectively, our results indicated that FBXW7 knockout promoted proliferation and TMZ tolerance of GBM cells.

Exosomal transfer of miR-25-3p increases $c-M y c$ and cyclin $E$ expression by regulating $F B X W 7$. FBXW7 is a key regulator of the cell cycle and cancer stemness (28). Accumulating evidence has indicated that c-Myc and cyclin E are downstream targets of FBXW7 (30-32). This was also evaluated in the present study. It was proven that miR-25-3p overexpression decreased FBXW7 expression, and simultaneously increased the expression of c-Myc and cyclin E in the U87 and A172 cells (Fig. 7A and B). These results indicated that the enforced expression of miR-25-3p promoted c-Myc and cyclin E expression by decreasing FBXW7 expression. Thus, it was 

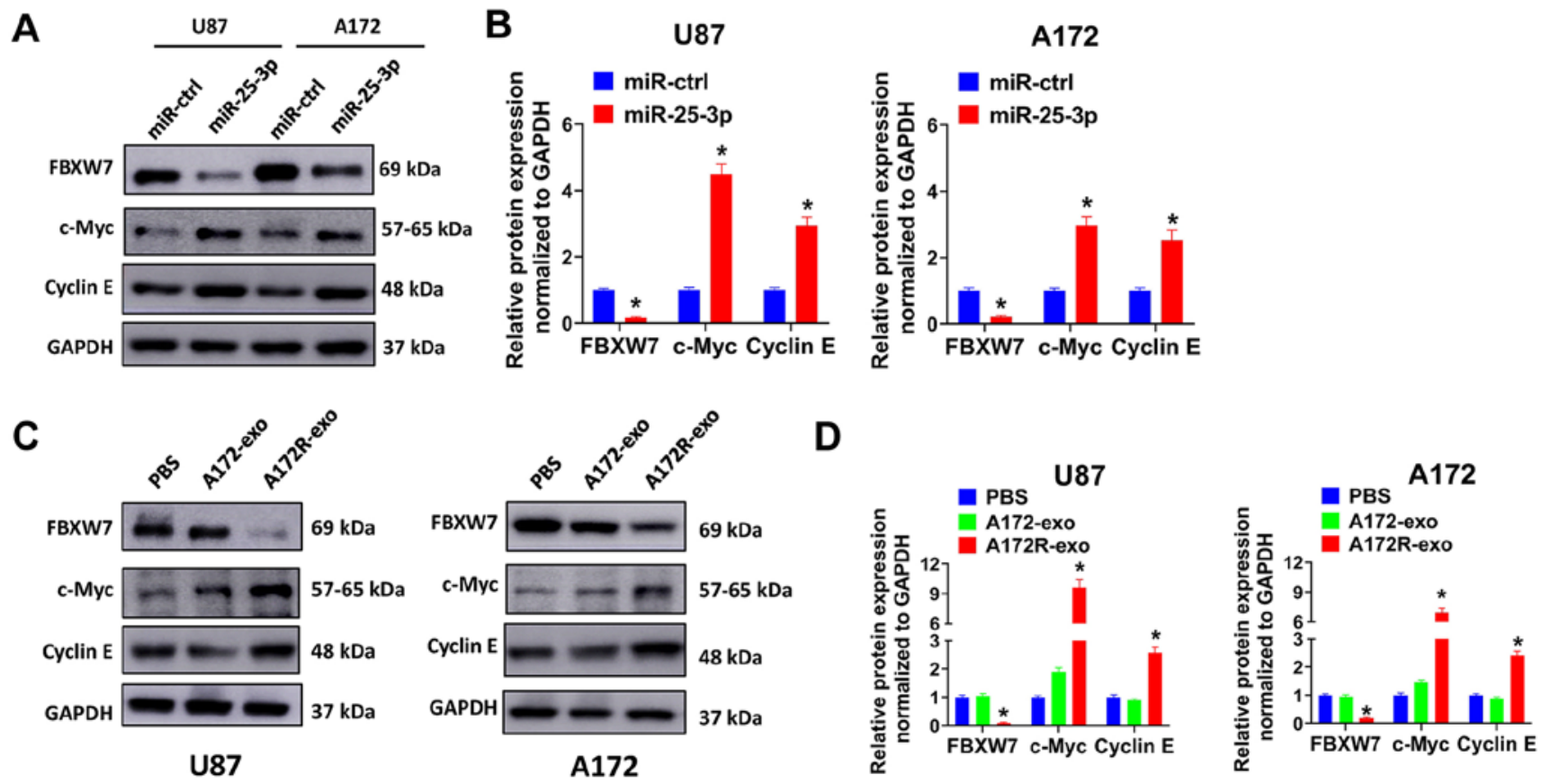

Figure 7. Exosomal transfer of miR-25-3p increases c-Myc and cyclin E expression by regulating FBXW7. (A and B) U87 or A172 cells were transduced with miR-25-3p expression lentivirus or miR-ctrl, and the (A) protein expression of FBXW7, c-Myc and cyclin E was then evaluated by western blot analysis. (B) Relative protein expression levels normalized to GAPDH are shown. (C and D) U87 or A172 cells were treated with A172R-exo (50 $\mu \mathrm{g} / \mathrm{ml}), \mathrm{A} 172$-exo (50 $\mu \mathrm{g} / \mathrm{ml})$ or an equal volume of PBS, and (C) the protein expression of FBXW7, c-Myc and cyclin E was then evaluated by western blot analysis. (D) Relative protein expression levels normalized to GAPDH are shown. All experiments were performed in triplicate. "P $\leq 0.05$. FBXW7, F-box and WD repeat domain containing 7.

hypothesized exosomes derived from TMZ-resistant A172R cells may also promote c-Myc and cyclin E expression. As was expected, the U87 and A172 cells treated with A172R-exo exhibited a decreased expression of FBXW7 and an increased expression of c-Myc and cyclin E compared with the cells treated with A172-exo or PBS (Fig. 7C and D). These results proved that the exosomal transfer of miR-25-3p increased c-Myc and cyclin E expression by regulating FBXW7 in GBM cells, and this may be one of the mechanisms involved in the promotion of the proliferation and TMZ resistance of GBM cells by exosomal miR-25-3p.

\section{Discussion}

Exosomes play a key role in the tolerance of GBM to TMZ. The exosomal transfer of proteins and RNAs may confer TMZ resistance to recipient cells. For example, the exosomal transfer of PTPRZ1-MET fusion protein has been shown to facilitate the migration, invasion, neurosphere growth and TMZ resistance of GBM cells (33). CircNFIX has also been shown to be increased in serum samples of patients with TMZ-resistant GBM. The exosomal transfer of CircNFIX enhances the migration, invasion and inhibits apoptosis of GBM cells under TMZ exposure (34). As previously demonstrated, lncRNA HOTAIR is overexpressed in TMZ-resistant glioma cells and promotes proliferation, metastasis and epithelial-mesenchymal transition. The exosomal transfer of HOTAIR induces TMZ resistance in target cells via the miR-519a/RRM1 axis (35). miR-221 expression has been shown to be increased in tissue samples and exosomes of glioma patients. Exosomal miR-221 promotes tumorigenesis and TMZ resistance of glioma cells by targeting DNM3 (36). In the present study, a TMZ-resistant
GBM cell line (A172R) was established. It was found that exosomes of A172R cells (A172R-exo) promoted the growth and TMZ resistance of sensitive GBM cells. Moreover, miRNA sequencing data for A172R-exo identified miR-25-3p as a miRNA associated with TMZ resistance. The depletion of miR-25-3p in A172R-exo partially abrogated the effects caused by A172R-exo transfer. These data proved that the exosomal transfer of miR-25-3p may confer TMZ resistance to recipient cells.

The present study has some potential limitations which should be mentioned. Only one TMZ-resistant cell line was established and used to verify the effects of exosomes and the exosomal miR-25-3p of this TMZ-resistant cell line. The data would be more valid if the results could be duplicate in other TMZ-resistant cell lines. Moreover, the results were mainly conducted on GBM cell lines in vitro and nude mice in vivo; thus, there are a lack of data on patient samples, such as patient-derived tumor xenografts or tumor cells. Finally, the number of patients in the present study were small, and the association of exosomal miR-25-3p with TMZ resistance and the prognosis of patients with GBM was not fully elucidated.

miR-25-3p is overexpressed and functions as an oncogene in various cancers, including GBM. For example, as previously demonstrated, a high level of miR-25-3p facilitates the malignant progression of pancreatic cancer cells by decreasing PHLPP2 and subsequently activating AKT/p70S6K signaling (16). Moreover, miR-25-3p has been shown to facilitate the proliferation and tumor xenograft growth of breast cancer cells by suppressing BTG2 and indirectly activating AKT and ERK/MAPK signaling (17). miR-25 has been shown to be evidently overexpressed in 
astrocytoma and GBM cells lines. The ectopic expression of miR-25 has also been shown to increase the proliferation and invasion of GBM cells by targeting NEFL (27). In another study, miR-25-3p was reported to regulate the growth and migration of glioma cells by targeting FBXW7 and DKK3 (26). In the present study, it was found that the enforced expression of miR-25-3p promoted the proliferation and TMZ resistance of GBM cells, indicating that miR-25-3p functioned as an oncogene in GBM, in accordance with the findings of previous studies $(26,27)$. It was also revealed that miR-25-3p was overexpressed in the serum of TMZ-treated patients with GBM and exosomes of TMZ-resistant GBM cells. Indeed, circulating miR-25-3p may be a predictor of prognosis in cancers, such as osteosarcoma (37) and gastric cancer (38). Ebrahimkhani et al (39) reported that miR-25-3p expression was increased in the serum of patients with GBM, suggesting that miR-25-3p was a potential diagnostic marker for GBM. The present study found that the exosomal delivery of miR-25-3p spread TMZ tolerance to other sensitive GBM cells. Notably, the exosomal delivery of miR-25-3p has exhibited cancer-promoting activity in other types of cancers as well. For example, in a previous study, the exosomal delivery of miR-25-3p induced a pre-metastatic nice by inhibiting KLF2 and KLF4, thus facilitating vascular permeability and the angiogenesis of colorectal cancer (40). In liposarcoma, miR-25-3p has been shown to promote the IL-6 production of tumor-associated macrophages, thus stimulating the proliferation and metastasis of liposarcoma cells (41). These studies and the findings of the present study indicate an important function of exosomal miR-25-3p in the tumorigenesis and drug resistance of cancer.

FBXW7 is a part of the Skp1-Cullin1-F-box (SCF) complex. The SCF complex plays a role in the degradation of oncoproteins, such as c-Myc, Notch, cyclin E and c-Jun (30). FBXW7 functions as a tumor suppressor in GBM. For example, FBXW7 has been proven to be negatively associated with glioma histology and positively with patient survival, while FBXW7 knockdown regulates proliferation, metastasis and the TMZ resistance of GBM cells (29). FBXW7 has been shown to be downregulated in GBM tissues, and the overexpression of FBXW7 suppresses the proliferation of GBM cells in vitro (42). In a previous study, in a genetic-engineered mouse model, p53 mutation was proven to promote gliomagenesis by inhibiting FBXW7 expression, thus increasing $\mathrm{c}-\mathrm{Myc}$ expression and protecting against $\mathrm{c}-\mathrm{Myc}$ induced apoptosis (43). The present study revealed that FBXW7 was targeted by miR-25-3p. FBXW7 knockdown facilitated the proliferation and TMZ tolerance of GBM cells. Moreover, c-Myc and cyclin E expression levels were increased by FBXW7 knockdown. The results proved that the effects of the exosomal transfer of miR-25-3p may be due to the inhibition of the tumor suppressor, FBXW7, thus increasing the levels of oncoproteins, such as c-Myc and cyclin E. Indeed, there are several studies demonstrating that FBXW7 is a direct target of miR-25-3p. For example, Wang et al (44) reported that miR-25 regulates cardiomyocyte growth by inhibiting FBXW7. In prostatic small cell neuroendocrine carcinoma, p53 mutation has been proven to inhibit FBXW7 expression by upregulating miR-25 (45). mR-25 has also been reported to exert cancer-promoting effects in esophageal squamous cell carcinoma and glioma by suppressing FBXW7 $(26,46)$. Although it is well-established that FBXW7 is targeted by miR-25-3p, the novelty of the present study is that it mainly focuses on the function of exosomes of TMZ-resistant GBM cells. The present study proved that the exosomal transfer of miR-25-3p may spread TMZ resistance to sensitive GBM cells, thus revealing an important role of tumor-derived exosomes in drug resistance. Moreover, the present study also demonstrated, for the first time, to the best of our knowledge, that exosomal miR-25-3p was involved in acquired TMZ resistance in GBM.

In conclusion, the present study found that exosomes of TMZ-resistant GBM cells promoted the proliferation and spread TMZ resistance to other GBM cells. miRNA sequencing data identified miR-25-3p as a significantly upregulated exosomal miRNA. The depletion of exosomal miR-25-3p partially abolished the effects induced by exosomes derived from TMZ-resistant GBM cells. Moreover, miR-25-3p overexpression promoted the proliferation and TMZ resistance of GBM cells in vitro and in vivo. In addition, FBXW7 was proven to be a direct target of miR-25-3p. The knockdown of FBXW7 increased the proliferation and TMZ resistance of GBM cells. Furthermore, the exosomal transfer of miR-25-3p decreased c-Myc and Cyclin E expression by inhibiting FBXW7. The results of the present study provide new insight into exosomal miRNAs in the acquired TMZ resistance of GBM cells. Exosomal miR-25-3p may thus be a potential prognostic marker for patients with GBM.

\section{Acknowledgements}

Not applicable.

\section{Funding}

No funding was received.

\section{Availability of data and materials}

The datasets used and/or analyzed during the current study are available from the corresponding author on reasonable request.

\section{Authors' contributions}

All authors guarantee the integrity of the entire study. The experiments were conducted by JW, TL and BW. Clinical analyses were conducted by BW. Data were analyzed by TL. The manuscript was prepared and reviewed by JW. All authors have read and approved the manuscript. JW and TL confirm the authenticity of all the raw data.

\section{Ethics approval and consent to participate}

All patients and healthy donors signed the written informed consents. The collection and use of serum samples were approved by the Ethics Committee of the Henan Provincial People's Hospital [SYXK (Yu) 2018-0004]. The animal experiments were reviewed and approved by the Animal Care and Experimental Committee of Henan Provincial People's Hospital. 


\section{Patient consent for publication}

Not applicable.

\section{Competing interests}

The authors declare that they have no competing interests.

\section{References}

1. Louis DN, Ohgaki H, Wiestler OD, Cavenee WK, Burger PC, Jouvet A, Scheithauer BW and Kleihues P: The 2007 WHO classification of tumours of the central nervous system. Acta Neuropathol 114: 97-109, 2007.

2. Ostrom QT, Gittleman H, Fulop J, Liu M, Blanda R, Kromer C, Wolinsky Y, Kruchko C and Barnholtz-Sloan JS: CBTRUS statistical report: Primary brain and central nervous system tumors diagnosed in the United States in 2008-2012. Neuro Oncol 17 Suppl 4(Suppl 4): iv1-iv62, 2015.

3. Stupp R, Hegi ME, Mason WP, van den Bent MJ, Taphoorn MJ, Janzer RC, Ludwin SK, Allgeier A, Fisher B, Belanger K, et al: Effects of radiotherapy with concomitant and adjuvant temozolomide versus radiotherapy alone on survival in glioblastoma in a randomised phase III study: 5-year analysis of the EORTC-NCIC trial. Lancet Oncol 10: 459-466, 2009.

4. Stupp R, Mason WP, van den Bent MJ, Weller M, Fisher B, Taphoorn MJ, Belanger $\mathrm{K}$, Brandes AA, Marosi C, Bogdahn U, et al: Radiotherapy plus concomitant and adjuvant temozolomide for glioblastoma. N Engl J Med 352: 987-996, 2005.

5. Davis ME: Glioblastoma: Overview of disease and treatment Clin J Oncol Nurs 20(Suppl 5): S2-S8, 2016.

6. Kalluri R and LeBleu VS: The biology, function, and biomedical applications of exosomes. Science 367: eaau6977, 2020.

7. Valadi H, Ekstrom K, Bossios A, Sjostrand M, Lee JJ and Lotvall JO: Exosome-mediated transfer of mRNAs and microRNAs is a novel mechanism of genetic exchange between cells. Nat Cell Biol 9: 654-659, 2007.

8. Mashouri L, Yousefi H, Aref AR, Ahadi AM, Molaei F and Alahari SK: Exosomes: Composition, biogenesis, and mechanisms in cancer metastasis and drug resistance. Mol Cancer 18: $75,2019$.

9. Skog J, Wurdinger T, van Rijn S, Meijer DH, Gainche L, Sena-Esteves M, Curry WT Jr, Carter BS, Krichevsky AM and Breakefield XO: Glioblastoma microvesicles transport RNA and proteins that promote tumour growth and provide diagnostic biomarkers. Nat Cell Biol 10: 1470-1476, 2008.

10. Pace KR, Dutt R and Galileo DS: Exosomal L1CAM stimulates glioblastoma cell motility, proliferation, and invasiveness. Int J Mol Sci 20: 3982, 2019.

11. Zhang Z, Yin J, Lu C, Wei Y, Zeng A and You Y: Exosomal transfer of long non-coding RNA SBF2-AS1 enhances chemoresistance to temozolomide in glioblastoma. J Exp Clin Cancer Res 38: 166, 2019

12. Sun Z, Shi K, Yang S, Liu J, Zhou Q, Wang G, Song J, Li Z, Zhang $Z$ and Yuan W: Effect of exosomal miRNA on cancer biology and clinical applications. Mol Cancer 17: 147, 2018.

13. Wang B, Mao JH, Wang BY, Wang LX, Wen HY, Xu LJ, Fu JX and Yang H: Exosomal miR-1910-3p promotes proliferation, metastasis, and autophagy of breast cancer cells by targeting MTMR3 and activating the NF-kB signaling pathway. Cancer Lett 489: 87-99, 2020

14. Ghaemmaghami AB, Mahjoubin-Tehran M, Movahedpour A Morshedi K, Sheida A, Taghavi SP, Mirzaei H and Hamblin MR: Role of exosomes in malignant glioma: MicroRNAs and proteins in pathogenesis and diagnosis. Cell Commun Signal 18: 120, 2020.

15. Yin J, Zeng A, Zhang Z, Shi Z, Yan W and You Y: Exosomal transfer of miR-1238 contributes to temozolomide-resistance in glioblastoma. EBioMedicine 42: 238-251, 2019.

16. Zhang J, Bai R, Li M, Ye H, Wu C, Wang C, Li S, Tan L, Mai D, Li G, et al: Excessive miR-25-3p maturation via $\mathrm{N}^{6-}$ methyladenosine stimulated by cigarette smoke promotes pancreatic cancer progression. Nat Commun 10: 1858, 2019.

17. Chen H, Pan H, Qian Y, Zhou W and Liu X: MiR-25-3p promotes the proliferation of triple negative breast cancer by targeting BTG2. Mol Cancer 17: 4, 2018.
18. Zhang L, Tong Z, Sun Z, Zhu G, Shen E and Huang Y: MiR-25-3p targets PTEN to regulate the migration, invasion, and apoptosis of esophageal cancer cells via the PI3K/AKT pathway. Biosci Rep 40: BSR20201901, 2020.

19. Ning L, Zhang M,Zhu Q, Hao F, Shen W and Chen D: MiR-25-3p inhibition impairs tumorigenesis and invasion in gastric cancer cells in vitro and in vivo. Bioengineered 11: 81-90, 2020.

20. Rao HC, Wu ZK, Wei SD, Jiang Y, Guo QX, Wang JW, Chen CX and Yang HY: MiR-25-3p serves as an oncogenic MicroRNA by downregulating the expression of merlin in osteosarcoma. Cancer Manag Res 12: 8989-9001, 2020.

21. Ebert MS and Sharp PA: MicroRNA sponges: Progress and possibilities. RNA 16: 2043-2050, 2010.

22. Livak KJ and Schmittgen TD: Analysis of relative gene expression data using real-time quantitative PCR and the 2(-Delta Delta C(T)) Method. Methods 25: 402-408, 2001

23. Lu Y, Zhao X, Liu Q, Li C, Graves-Deal R, Cao Z, Singh B, Franklin JL, Wang J, Hu H, et al: lncRNA MIR100HG-derived miR-100 and miR-125b mediate cetuximab resistance via Wnt/ $\beta$-catenin signaling. Nat Med 23: 1331-1341, 2017.

24. Lee SY: Temozolomide resistance in glioblastoma multiforme. Genes Dis 3: 198-210, 2016.

25. Kim DK, Nishida H, An SY, Shetty AK, Bartosh TJ and Prockop DJ: Chromatographically isolated CD63+CD81+ extracellular vesicles from mesenchymal stromal cells rescue cognitive impairments after TBI. Proc Natl Acad Sci USA 113: 170-175, 2016.

26. Peng G, Yang C, Liu Y and Shen C: MiR-25-3p promotes glioma cell proliferation and migration by targeting FBXW7 and DKK3. Exp Ther Med 18: 769-778, 2019.

27. Peng G, Yuan X, Yuan J, Liu Q, Dai M, Shen C, Ma J, Liao Y and Jiang W: MiR-25 promotes glioblastoma cell proliferation and invasion by directly targeting NEFL. Mol Cell Biochem 409: 103-111, 2015.

28. Davis RJ, Welcker M and Clurman BE: Tumor suppression by the Fbw7 ubiquitin ligase: Mechanisms and opportunities. Cancer Cell 26: 455-464, 2014

29. Lin J, Ji A, Qiu G, Feng H, Li J, Li S, Zou Y, Cui Y, Song C, He H and Lu Y: FBW7 is associated with prognosis, inhibits malignancies and enhances temozolomide sensitivity in glioblastoma cells. Cancer Sci 109: 1001-1011, 2018

30. Sailo BL, Banik K, Girisa S, Bordoloi D, Fan L, Halim CE, Wang H, Kumar AP, Zheng D, Mao X, et al: FBXW7 in cancer: What has been unraveled thus far? Cancers (Basel) 11: 246, 2019

31. Ibusuki M, Yamamoto Y, Shinriki S, Ando Y and Iwase H: Reduced expression of ubiquitin ligase FBXW7 mRNA is associated with poor prognosis in breast cancer patients. Cancer Sci 102: 439-445, 2011.

32. Iwatsuki M, Mimori K, Ishii H, Yokobori T, Takatsuno Y, Sato T, Toh H, Onoyama I, Nakayama KI, Baba H and Mori M: Loss of FBXW7, a cell cycle regulating gene, in colorectal cancer: Clinical significance. Int J Cancer 126: 1828-1837, 2010.

33. Zeng AL, Yan W, Liu YW, Wang Z, Hu Q, Nie E, Zhou X, Li R, Wang XF, Jiang T and You YP: Tumour exosomes from cells harbouring PTPRZ1-MET fusion contribute to a malignant phenotype and temozolomide chemoresistance in glioblastoma. Oncogene 36: 5369-5381, 2017.

34. Ding C, Yi X, Wu X, Bu X, Wang D, Wu Z, Zhang G, Gu J and Kang D: Exosome-mediated transfer of circRNA CircNFIX enhances temozolomide resistance in glioma. Cancer Lett 479: $1-12,2020$.

35. Yuan Z, Yang Z,Li W, Wu A, SuZ and Jiang B: Exosome-mediated transfer of long noncoding RNA HOTAIR regulates temozolomide resistance by miR-519a-3p/RRM1 axis in glioblastoma. Cancer Biother Radiopharm 24 Jul, 2020 (Epub ahead of print).

36. Yang JK, Yang JP, Tong J, Jing SY, Fan B, Wang F, Sun GZ and Jiao BH: Exosomal miR-221 targets DNM3 to induce tumor progression and temozolomide resistance in glioma. J Neurooncol 131: 255-265, 2017.

37. Fujiwara T, Uotani K, Yoshida A, Morita T, Nezu Y, Kobayashi E, Yoshida A,Uehara T,Omori T, Sugiu K, et al: Clinical significance of circulating miR-25-3p as a novel diagnostic and prognostic biomarker in osteosarcoma. Oncotarget 8: 33375-33392, 2017.

38. ZiaSarabi P, Sorayayi S, Hesari A and Ghasemi F: Circulating microRNA-133, microRNA-17 and microRNA-25 in serum and its potential diagnostic value in gastric cancer. J Cell Biochem 120: 12376-12381, 2019.

39. Ebrahimkhani S, Vafaee F, Hallal S, Wei H, Lee MYT, Young PE, Satgunaseelan L, Beadnall H, Barnett MH, Shivalingam B, et al: Deep sequencing of circulating exosomal microRNA allows non-invasive glioblastoma diagnosis. NPJ Precis Oncol 2: 28, 2018. 
40. Zeng Z, Li Y, Pan Y, Lan X, Song F, Sun J, Zhou K, Liu X, Ren X, Wang F, et al: Cancer-derived exosomal miR-25-3p promotes pre-metastatic niche formation by inducing vascular permeability and angiogenesis. Nat Commun 9: 5395, 2018.

41. Casadei L, Calore F, Creighton CJ, Guescini M, Batte K, Iwenofu OH, Zewdu A, Braggio DA, Bill KL, Fadda P, et al: Exosome-derived miR-25-3p and miR-92a-3p stimulate liposarcoma progression. Cancer Res 77: 3846-3856, 2017.

42. Hagedorn M, Delugin M, Abraldes I, Allain N, BelaudRotureau MA, Turmo M, Prigent C, Loiseau H, Bikfalvi A and Javerzat S: FBXW7/hCDC4 controls glioma cell proliferation in vitro and is a prognostic marker for survival in glioblastoma patients. Cell Div 2: 9, 2007.

43. Kim HS, Woolard K, Lai C, Bauer PO, Maric D, Song H, Li A, Kotliarova S, Zhang W and Fine HA: Gliomagenesis arising from Pten- and Ink4a/Arf-deficient neural progenitor cells is mediated by the p53-Fbxw7/Cdc4 pathway, which controls c-Myc. Cancer Res 72: 6065-6075, 2012 .
44. Wang B, Xu M, Li M, Wu F, Hu S, Chen X, Zhao L, Huang Z, Lan F, Liu D and Wang Y: MiR-25 promotes cardiomyocyte proliferation by targeting FBXW7. Mol Ther Nucleic Acids 19: 1299-1308, 2020.

45. Li Z, Sun Y, Chen X, Squires J, Nowroozizadeh B, Liang C and Huang J: p53 mutation directs AURKA overexpression via miR-25 and FBXW7 in prostatic small cell neuroendocrine carcinoma. Mol Cancer Res 13: 584-591, 2015.

46. Hua Y, Zhao K, Tao G, Dai C and Su Y: MiR-25 promotes metastasis via targeting FBXW7 in esophageal squamous cell carcinoma. Oncol Rep 38: 3030-3038, 2017.

This work is licensed under a Creative Commons Attribution-NonCommercial-NoDerivatives 4.0 International (CC BY-NC-ND 4.0) License. 